\title{
A method for calibration of the Hyperplastic Accelerated Ratcheting Model (HARM)
}

\author{
Abadie, C.N. ${ }^{1}$, Houlsby, G.T. ${ }^{2}$ and Byrne, B.W. ${ }^{2}$ \\ ${ }^{1}$ Department of Engineering Science, University of Cambridge \\ Trumpington Street, Cambridge CB2 1PZ, UK \\ (formerly Department of Engineering Science, University of Oxford) \\ ${ }^{2}$ Department of Engineering Science, University of Oxford \\ Parks Road, Oxford OX1 3PJ, UK \\ Contact: cna24@cam.ac.uk (corresponding author), guy.houlsby@eng.ox.ax.uk, \\ byron.byrne@eng.ox.ac.uk
}

\begin{abstract}
This paper presents an analytical methodology for calibration of the Hyperplastic Accelerated Ratcheting Model (HARM) [3], based on a closed-form expression for the accumulation of ratcheting strain with cyclic history. The proposed method requires the fit of one test response and of a few continuous cyclic tests. The initial motivation for this work is the calibration of models for the design of piles subjected to longterm cyclic lateral loading, and the test results from Abadie, Byrne [1], [2] are used for calibration and proofing of the model. Nevertheless, the method is applicable to other problems of similar behaviour.
\end{abstract}

KEYWORDS: Cycling, Hysteresis, Plasticity, Ratcheting, Calibration

\section{Introduction}

There are numerous problems in engineering, where a system or material subjected to cyclic loading will exhibit accumulated permanent deformation with cycle number. For many problems, the worst scenario occurs when the response never reaches an accommodated or adapted state and keeps accumulating permanent deformation, even at very large cycle number. In this case, called ratcheting, prediction of the response, and in particular the residual deformation after a series of load packets, is crucial. However, very few models are capable of doing so.

The most common modelling approach currently used to capture ratcheting is based on the ArmstrongFrederick (AF) model [4-6], with many variants being published and principally used for modelling alloys 
(e.g. $[7,8])$. An example calibration of the AF model is described by Lemaitre and Chaboche [6]. Houlsby et al. [3] provide a review of the AF model, and the discuss the rationale for not pursuing such methodology further, in particular for the case where the cyclic loading response conforms approximately to the extended Masing rules [9], while accumulating permanent ratcheting strain under cyclic loading. One of the key reasons is that for the AF model it is difficult to calibrate both the initial loading response and the accumulated ratcheting strain (e.g. $[6,7])$.

In geomechanics, and more specifically for pile foundation design, the effects of cyclic loading has long been captured using so-called "degradation methods", where the soil resistance is reduced by a factor that is either a constant (e.g. [10-13]) or a function of the number of cycles (e.g. $[14,15])$. More recent modelling uses similar approaches within 3D FE analysis [16]. However, these methods are largely empirical, and importantly, do not enable the full load-deflection response of the foundation to be determined. As a result, the response to realistic multi-amplitude cyclic loading, as well as the estimation of the ULS response following long-term cyclic history, is impossible to predict without the use of further assumptions such as linear cumulative damage ([17], [18]).

Houlsby et al. [3] present a new approach for modelling of ratcheting in a computationally efficient way, called the HARM model (Hyperplastic Accelerated Ratcheting Model). This model addresses the challenging issue of capturing accumulation of ratcheting strain with cycle number for a large number of cycles, while conforming approximately to the extended Masing rules [9]. Such methodology enables the entire load-deflection response to be predicted incrementally, and as a direct consequence the response to any load history of varying amplitude can be determined. An example calculation, including a set of parameter values, is also provided in [3], with no further explanation on calibration methodology. However, for use in design, calibration process is vital and the work presented here provides additional development on how this can be achieved.

The primary motivation behind this work is the design of offshore wind turbine monopiles when subjected to many cycles of large horizontal loads. In this case, design guidance, such as by DNV, explicitly advises 
to address permanent deformations due to cyclic load history (e.g. [13] , Section 10.3.2.6, p. 173) and rigorous design also needs to assess (a) how the natural frequency is affected by cyclic loading, (b) whether the ULS capacity has changed, and (c) how the fatigue life developed during the cyclic process. Unfortunately, no design standard yet provides detailed guidelines as to how this issue should be addressed within design codes and practice. Most of the methods proposed thus far make use of empirical methods, similar to those described above, where empirical coefficients are employed to alter the monotonic response according to the number of cycles undertaken by the foundation during its lifetime, e.g. $[16,19,20]$ and do not provide information on items (a), (b) and (c) for structural design.

This paper describes theoretical developments for HARM that enable to (i) accommodate ratcheting behaviour accurately and (ii) calibrate the model to address the load-deflection relationship for a pile under lateral loading. The experimental test results presented in Abadie, Byrne [1, 3] are used as an impetus for the work, and the model accuracy is evaluated by comparing the predictions with the test results. The modelling approach, specific to offshore wind turbine structures, is explained at the beginning of the paper (Section 2). However, the mathematical developments are expressed in such a way that they could be applied to other mechanical problems, providing that they exhibit a similar hysteretic and ratcheting response.

\section{Pre-requisite modelling}

\subsection{Objective cyclic behaviour: experimental evidence}

Results from laboratory tests provide fundamental insights on the foundation behaviour that determine the form of development of theoretical and numerical models. The work published by Abadie, Byrne [1, 3] and Abadie [21] involves laboratory floor model tests, scaled to represent a full-scale wind-turbine monopile in drained sand, and elaborates clearly the important effects that need to be accounted for in detailed modelling:

(0) Increase in accumulated ratcheting deformation due to cyclic loading at non-zero mean stress while conforming approximately to Masing behaviour [9] (kinematic hardening), 
(1) Decrease of ratcheting rate with cycle number that never decays to zero, at least for the first $1,000,000$ cycles of continuous cyclic loads at a maximum load above $20 \%$ ultimate capacity,

(2) Dependence of the ratcheting behaviour on the cyclic load magnitude,

(3) Change in hysteresis loop shape, including an increase in secant stiffness and reduction of the hysteresis loop area, mostly during the first 50 cycles of a given load magnitude.

Item (0) is identified as the fundamental feature describing the pile response, while (1) to (3) characterise the ratcheting behaviour in more detail. These form the targeted features that this paper aims to capture in modelling and will be referred to as principles $(0)$ to $(3)$ in the following. The tests considered here were performed in dry sand, reproducing drained loading offshore. In these conditions, Abadie et al. [1] showed that rate effects can be neglected, allowing a focus on the development of a modelling method for ratcheting. This is a step towards a comprehensive modelling framework for offshore monopile design. The challenging case of water-saturated soils under partial drainage conditions resulting in transient pore pressures is not considered here.

A monotonic test (MCo) and the three long-term one-way continuous cyclic tests (CMLT1,2 and 3) from this work are used for calibration. The remaining tests, as well as the two critical tests from Abadie, Byrne [2], which involve more complicated load scenarios, are finally used for validation of the calibrated model. A summary of the tests used in this paper is provided in Table 1 and the detail of the testing set-up, as well as the limitations of the testing conditions, are discussed in $[1,21]$.

\subsection{Hyperplastic Accelerated Ratcheting Model (HARM)}

The HARM Model used in this paper is rooted in the "hyperplasiticity" framework, initially introduced by Ziegler $[22,13]$ and described in detail by Houlsby and Puzrin [24]. This approach enables description of non-linear behaviour of materials within a rigorous mathematical framework that enforces the model to be thermodynamically acceptable. The HARM extension enables this approach to capture the phenomenon of ratcheting under very many cycles, as observed during cyclic loading of rigid piles for offshore wind turbine applications [3]. The model is based on the multi-surface kinematic hardening 
Table 1. Selected test from [1] and [2] for proofing of the calibrated HARM model (cyclic load packet described in terms of Cycle number $x$ (load magnitude $\zeta_{b}$, Equation 14))

\begin{tabular}{|c|c|}
\hline Test No. & Loading description \\
\hline \multicolumn{2}{|c|}{ Monotonic test } \\
\hline MCo & Continuous monotonic loading \\
\hline \multicolumn{2}{|c|}{ Continuous cyclic tests followed by monotonic reload } \\
\hline CMLT1 & $100,000 \times\left(\zeta_{b}=0.31\right)$ \\
\hline CMLT2 & $100,000 \times\left(\zeta_{b}=0.42\right)$ \\
\hline CMLT3 & $100,000 \times\left(\zeta_{b}=0.47\right)$ \\
\hline CMC1 & $1 \times\left(\zeta_{b}=0.42\right)$ \\
\hline CMC2 & $10 \times\left(\zeta_{b}=0.42\right)$ \\
\hline CMC3 & $100 \times\left(\zeta_{b}=0.42\right)$ \\
\hline CMC4 & $1,000 \times\left(\zeta_{b}=0.42\right)$ \\
\hline CMC5 & $10,000 \times\left(\zeta_{b}=0.42\right)$ \\
\hline \multicolumn{2}{|c|}{ Multi-amplitude cyclic loading tests } \\
\hline H1IM & $\begin{array}{l}1 \times\left(\zeta_{b}=0.25\right)-1 \times\left(\zeta_{b}=0.34\right)-1 \times\left(\zeta_{b}=0.39\right)-1 \times\left(\zeta_{b}=0.50\right)-1 \times\left(\zeta_{b}=0.59\right)-\left(\zeta_{b}=0.70\right)-1 \times \\
\left(\zeta_{b}=0.86\right)\end{array}$ \\
\hline MALL1 & $100 \times\left(\zeta_{b}=0.48\right)-10 \times\left(\zeta_{b}=0.59\right)-1 \times\left(\zeta_{b}=0.69\right)$ \\
\hline MALL2 & $1 \times\left(\zeta_{b}=0.69\right)-100 \times\left(\zeta_{b}=0.48\right)-10 \times\left(\zeta_{b}=0.59\right)$ \\
\hline MALL3 & $10 \times\left(\zeta_{b}=0.59\right)-1 \times\left(\zeta_{b}=0.69\right)-100 \times\left(\zeta_{b}=0.48\right)$ \\
\hline MASL1 & $1000 \times\left(\zeta_{b}=0.30\right)-100 \times\left(\zeta_{b}=0.48\right)-1 \times\left(\zeta_{b}=0.69\right)$ \\
\hline MASL2 & $100 \times\left(\zeta_{b}=0.48\right)-1 \times\left(\zeta_{b}=0.69\right)-1000 \times\left(\zeta_{b}=0.30\right)$ \\
\hline MASL3 & $1 \times\left(\zeta_{b}=0.69\right)-100 \times\left(\zeta_{b}=0.48\right)-1000 \times\left(\zeta_{b}=0.30\right)$ \\
\hline MA1 & $\begin{array}{l}900 \times\left(\zeta_{b}=0.31\right)-100 \times\left(\zeta_{b}=0.51\right)-1 \times\left(\zeta_{b}=0.72\right)-100 \times\left(\zeta_{b}=0.51\right)-900 \times\left(\zeta_{b}=0.31\right)-100 \times \\
\left(\zeta_{b}=0.51\right)-1 \times\left(\zeta_{b}=0.72\right)-100 \times\left(\zeta_{b}=0.51\right)-9,500 \times\left(\zeta_{b}=0.31\right)\end{array}$ \\
\hline MA2 & $\begin{array}{l}1,000 \times\left(\zeta_{b}=0.31\right)-1 \times\left(\zeta_{b}=0.41\right)-500 \times\left(\zeta_{b}=0.31\right)-1 \times\left(\zeta_{b}=0.45\right)-500 \times\left(\zeta_{b}=0.31\right)-1 \\
\times\left(\zeta_{b}=0.49\right)-500 \times\left(\zeta_{b}=0.31\right)-1 \times\left(\zeta_{b}=0.54\right)-500 \times\left(\zeta_{b}=0.31\right)-1 \times\left(\zeta_{b}=0.6\right)-500 \times \\
\left(\zeta_{b}=0.31\right)-1 \times\left(\zeta_{b}=0.62\right)-500 \times\left(\zeta_{b}=0.31\right)-1 \times\left(\zeta_{b}=0.66\right)-6,500 \times\left(\zeta_{b}=0.31\right)\end{array}$ \\
\hline
\end{tabular}

hyperplasticity framework originally proposed by Puzrin and Houlsby $[25,15]$, and therefore, complies with kinematic hardening rules (also called Masing rules, $[9,27])$. It is supplemented by a ratcheting element (Figure 1(a)) that produces accumulation of deformation with cycling while still exhibiting approximate Masing behaviour, as shown in Figure 1(b). The model is applicable to rate-independent and rate-dependent materials. However, Abadie, Byrne [1] show that there is no evidence of strain rate affecting the pile response, and therefore, the rate-independent version of the model is used in the following. Alternatively, the rate-dependent form can be employed, with use of an appropriately small value of the viscosity $\mu$. 
Houlsby, Abadie [3] present both a "series" and "parallel" version of the HARM model, which both lead to a similar hysteretic and ratcheting behaviour, and in some cases, are equivalent (see Section 2.6). Ignoring the effects of ratcheting, Beuckelaers et al. [28] show that the series and parallel forms of the model result in an identical response for 1-directional loading. For 2-directional loading the responses are not identical, but are sufficiently close that they are within the bounds of experimental error. It would be extremely difficult to devise an experiment that would enable a definitive choice between the two models to be made. Beuckelaers et al. [28] therefore advise that the choice can be made on pragmatic grounds. They suggest using the series model for load-controlled problems, as it is computationally simpler for this case. Conversely, the parallel version is better suited for strain-control, and for implementation in most finite element codes. Assuming that the models are also very similar once ratcheting is included, the series form of the HARM model is used in the following analysis of load-controlled tests.

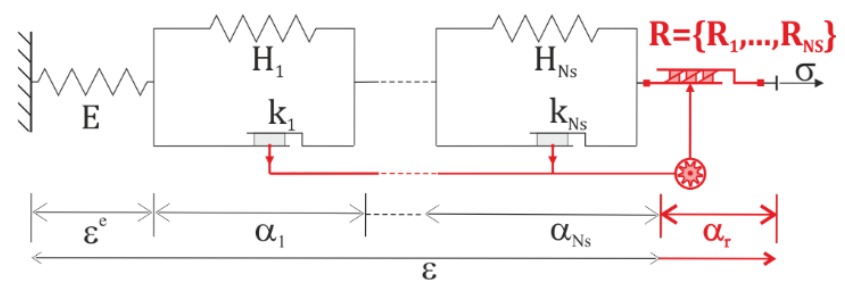

(a)

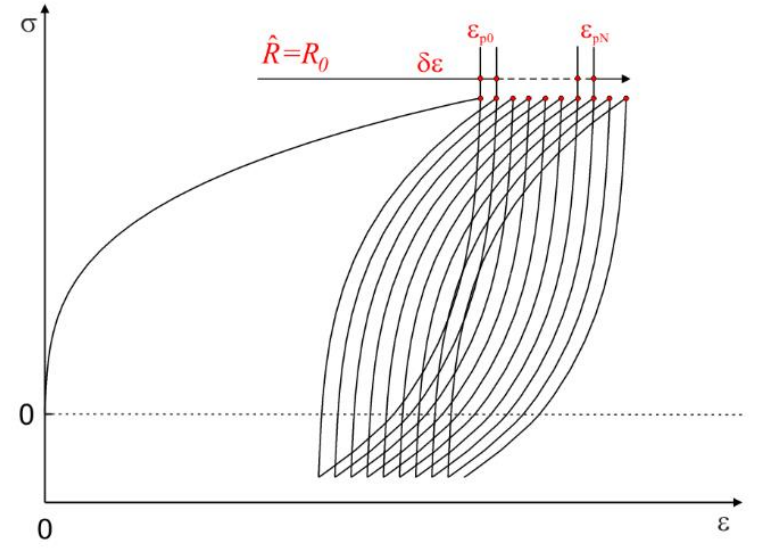

(b)

Figure 1. (a) Schematic representation and (b) Typical response continuous cyclic loading obtained with HARM (adapted from [3])

Mathematically, the constitutive behaviour is described in terms of "stress" and "strain" $(\sigma, \varepsilon)$ and is completely defined through the derivation of two scalar functions, here the energy function $f$ and the dissipation potential $d$ :

$f=\frac{E_{0}}{2}\left(\varepsilon-\sum_{n=1}^{N_{S}} \alpha_{n}-\alpha_{r}\right)^{2}+\sum_{n=1}^{N_{S}} \frac{H_{n}}{2} \alpha_{n}^{2}$ 
$d=\sum_{n=1}^{N_{S}} k_{n}\left|\dot{\alpha}_{n}\right|+|\sigma|\left|\dot{\alpha}_{r}\right|$

Equation 2

Where $E_{0}$ is the elastic modulus, the $H_{n}$ are the kinematic hardening moduli, the $k_{n}$ are the kinematic hardening surface strengths. The mathematical developments shown in Houlsby, Abadie [3] are formulated using a discrete summation of a large number $N_{S}$ of yield surfaces (summation symbols in Equation 1 and Equation 2). For very large number of $N_{S}$, this formulation can be developed one step further to describe the case of a continuous field of yield surfaces in a similar fashion as the work published in Puzrin and Houlsby [25]. This corresponds to replacing the discrete sums by integrals, which hence describes a perfectly smooth elastic-plastic transition (e.g., Figure 6(c) in [29]):

$$
\begin{aligned}
& f=\frac{E_{0}}{2}\left(\varepsilon-\int_{0}^{N_{S}} \hat{\alpha} d \eta-\alpha_{r}\right)^{2}+\int_{0}^{N_{S}} \frac{\hat{H}}{2} \hat{\alpha}^{2} d \eta \\
& d=\int_{0}^{N_{S}} \hat{k}|\dot{\hat{\alpha}}| d \eta+|\sigma|\left|\dot{\alpha}_{r}\right|
\end{aligned}
$$

$\eta$ is the internal coordinate that replaces the index $n$ and the set of internal variables $\left\{\alpha_{1}, \alpha_{2}, \ldots, \alpha_{N_{S}}\right\}$ becomes an internal function $\hat{\alpha}(\eta)$ of this coordinate. For large values of $N_{S}$, the two formulations are clearly equivalent, but the integral form is mathematically more rigorous and enables the developments described in this paper to be more concise. However, it is impractical for implementation purposes where a discrete formulation using a large value for $N_{S}$ is preferred. For computation application, a value of $N_{S}=$ 40 was adopted in this paper.

Using this formulation, the constraint defining the additional ratcheting strain $\alpha_{r}$ (Equation 13 in [3]), is defined through the constraint:

$c_{r}=\dot{\alpha}_{r}-S(\sigma) \int_{0}^{N_{S}} \hat{R}|\dot{\hat{\alpha}}| d \eta=0$ Equation 5

Where $\mathrm{S}()$ is the generalised signum function (see Notation). $\hat{R}$ is the family of ratcheting parameters of discretised form $R_{n}$ in Figure 1 (a). The $\hat{R}$ values are dimensionless fraction of unity that directly controls 
the amount of ratcheting strain. It is the most subtle of the quantities to accommodate and fit the ratcheting behaviour, and the objective of this paper is to demonstrate how to choose and calibrate their values. On the other hand, when $\forall n \in\left[1, N_{S}\right], R_{n} \equiv \hat{R}(\eta) \equiv 0$, all ratcheting effects are disabled and the resulting response corresponds to pure kinematic hardening.

Finally, the cyclic history is memorised through the accumulated ratcheting strain $\beta$ :

$c_{\beta}=\dot{\beta}-\left|\dot{\alpha}_{r}\right|$

Equation 6

$\beta$ is a state hardening variable that accounts for the change of behaviour with cyclic history. Applying standard methods of derivation within the hyperplasticity framework (see $[1-3,18]$ for methodology), the above yields to the expression of the yield surface:

$\sigma=\hat{k} S(\dot{\hat{\alpha}})+\hat{H} \hat{\alpha}$

Equation 7

And the incremental behaviour:

$$
\begin{aligned}
& d \varepsilon=\frac{d \sigma}{E}+\int_{0}^{N_{S}} d \hat{\alpha} d \eta+d \alpha_{r} \\
& d \alpha_{r}=S(\sigma) \int_{0}^{N_{S}} \hat{R}|d \alpha| d \eta \\
& d \beta=\left|d \alpha_{r}\right|
\end{aligned}
$$

Equation 10

Finally, the effect of ratcheting within any group of cycles of the same load magnitude and amplitude can be accelerated to save computation time in a rigorous and exact way. This is a very powerful feature of the model, achieved by multiplying Equation 9 by a factor $R_{f a c}$ that corresponds to the number of cycles to be "skipped" or "accelerated":

$$
d \alpha_{r}=R_{f a c} S(\sigma) \int_{0}^{N_{S}} \hat{R}|d \alpha| d \eta
$$

Implementation of Equation 7 to Equation 11 with a constant value for $\hat{R}$, reproduces ratcheting behaviour at a constant rate, while conforming approximately to Masing rules (Figure 1(b)) and therefore provides the fundamental behaviour of principle (0). 


\subsection{Macro-modelling approach and notation}

The objective of this paper is to model the response of an offshore wind turbine foundation to a combined moment and horizontal load, relevant to wind and wave loading on a monopile (Figure 2). The work done by this set of loads is equivalent to the work done by a load applied at an eccentricity $h_{e}=M / H$ from the ground level, which allows replacement of the coupled torsional $M-\theta$ and lateral $H-v_{G}$ reactions by a single lateral reaction $H-v_{T}$ for simplicity.

The modelling approach followed in this paper considers the macroscopic response $H-v_{T}$ of the pile and encapsulates its response through a single 0-D macro-element model at the ground surface (Figure 2). The authors appreciate that the model does not address the local soil behaviour down the pile when the pile is subjected to cyclic loading, which is a recognised limitation of the work. Clearly, the macroresponse of the pile is the integration of the local responses down the pile, and continuation of this work towards a comprehensive study linking the two would provide very useful insight for design. Nevertheless, the approach followed here provides the basis for implementation of soil-structure interaction macro element models within structural analysis packages, which are very widely used and of great importance to the offshore engineering industry. 


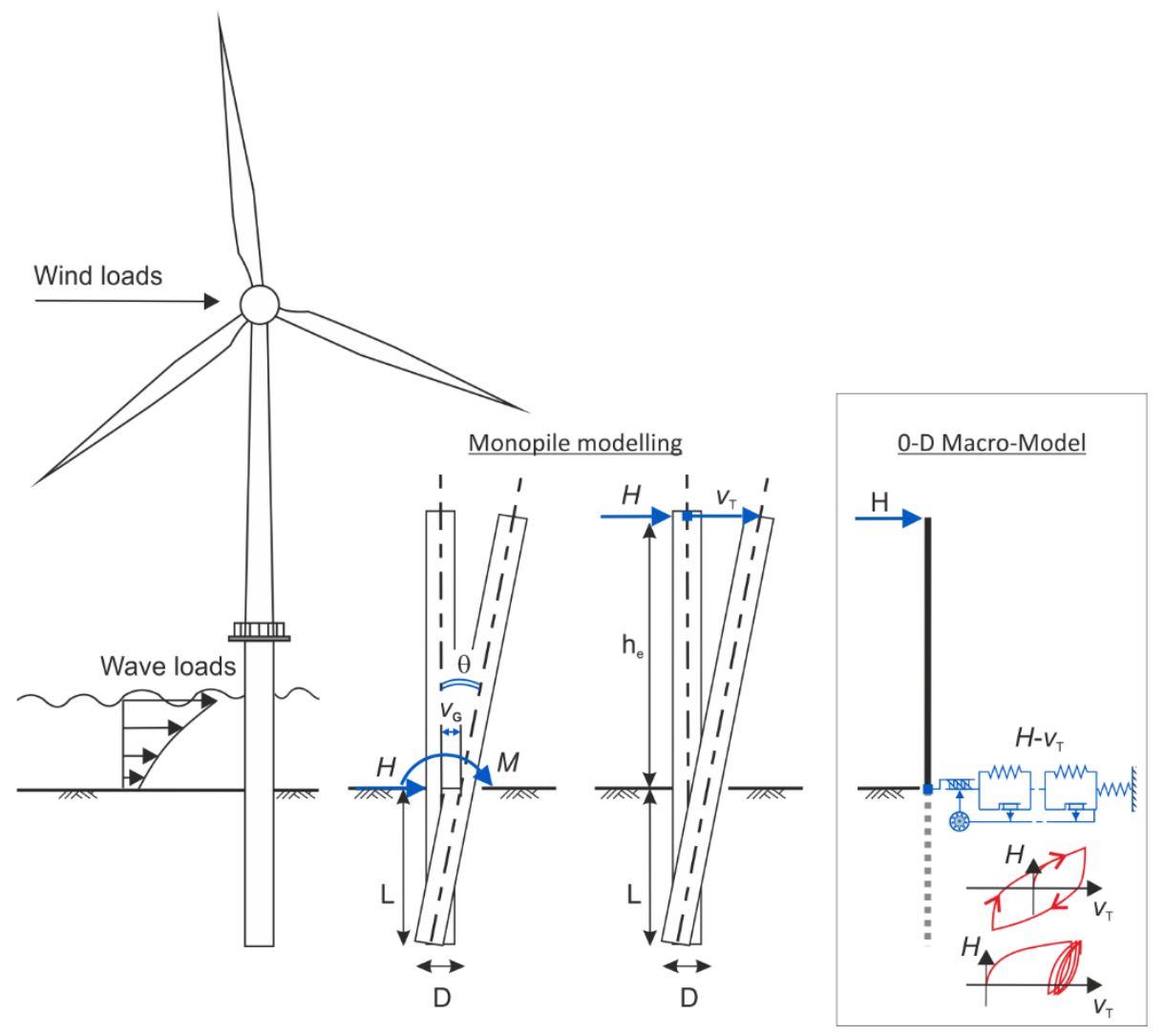

Figure 2. Schematic representation of monopile 0-D macro-modelling

For consistency with Houlsby, Abadie [3], the theoretical developments presented in the following are expressed in terms of stress $\sigma$ and strain $\varepsilon$, although in this particular application the "stress" stands for the lateral load on the pile and "strain" for the displacement at the point of load application. In addition, the lateral force and the pile top displacement are both normalised using the corresponding values at ultimate capacity $\left(H_{R}\right.$ and $\left.v_{T R}\right)$.

$\sigma=\frac{H_{t}}{H_{R}}$ Equation 12

$\varepsilon=\frac{v_{T}}{v_{T R}}$

Equation 13

For the pile in sand used by Abadie, Byrne [1] (with L=360mm, D=77mm), the values at pile top (load application level) are $H_{R}=M_{R} / h_{e}=78 \mathrm{~N}$ and $v_{T R}=19.51 \mathrm{~mm}=0.25 \times D$ at an eccentricity $h_{e}=$ $430 \mathrm{~mm}$, corresponding to a displacement of $v_{G} \approx 0.1 \times D$ at ground level. Using this notation, the normalised load magnitude $\zeta_{b}$ used in [19] and [1] and used to characterise cyclic loading test magnitude corresponds to: 
$\zeta_{b}=\sigma_{\max }=H_{t, \max } / H_{R}$

Careful consideration needs to be applied to the appropriate dimensions for all quantities, and in particular for the stiffness. Special attention is hence paid to notation and definitions of strain quantities, and Figure 3 displays the conventions used. In particular, the initial load-unload loop is indexed "0" to indicate that this cycle actually corresponds to the backbone curve. Subscripts " $p$ " and "m" are used to indicate (maximum) peak and minimum cyclic points respectively. Because of Masing rules, after any unload-reload cycle, at peak load, the accumulated strain is entirely caused by the accumulation of ratcheting strain (when there is no ratcheting, the loop is indeed closed), and hence:

$\varepsilon_{p N}-\varepsilon_{p 0}=\beta_{p N}-\beta_{p 0}$

Equation 15

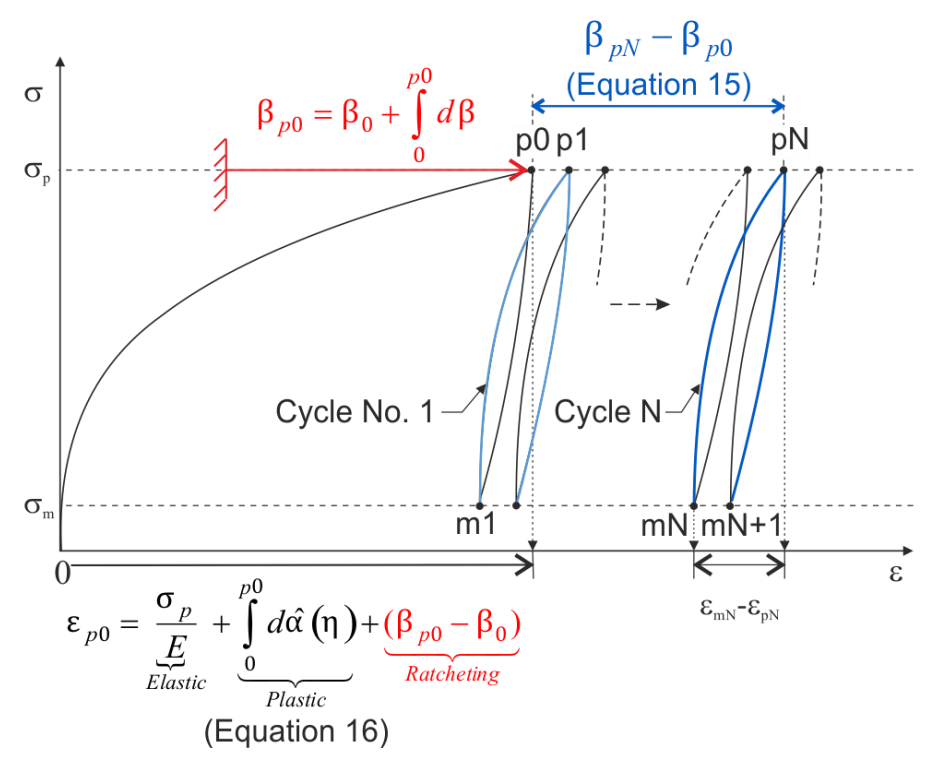

Figure 3. Notations for calibration of the model and illustration of hardening parameter

The calibration method proposed here requires the use of (i) a monotonic test and (ii) a small set of longterm cyclic tests (we use 3 tests here). The use of 1-way loading tests facilitates the mathematical developments shown below, and is hence preferred here. The long-term continuous cyclic test results presented in Abadie, Byrne [1] are not perfect one-way loading, as the experimental equipment did not allow to unload to zero, however, the minimum load is small enough to use this approximation.

Using this notation, Abadie, Byrne [1] demonstrate that a good fit of the backbone curve is achieved with: 
$\varepsilon=\frac{\sigma}{E_{o}}+\varepsilon_{p U}\left(\frac{\sigma}{k_{U}}\right)^{m_{h}} \quad$ on initial loading only

$E_{0}$ is the initial stiffness of the response, measured by the slope of the tangent to the origin. $k_{U}$ is the ultimate strength for the model sliders, which can conveniently be chosen equal to the ultimate load, and, according to Equation 12, is equal to 1 here. Similarly, $\varepsilon_{p u}$ is the ultimate monotonic plastic strain, equal to 1 according to Equation 13. Finally, $m_{h}$ is an empirical exponent that defines the shape of the curve of value $m_{h}=3[1,21]$

Equation 16 captures a continuing increase in strength with strain, which never reaches an ultimate limit state. In the particular case of application to a laterally loaded pile, this is not a problem, as the pile is never pushed to ultimate capacity and mostly performs within the non-linear plastic region.

For the case of laterally loaded monopiles subjected to 1-way loading, and the test results used in this paper, LeBlanc, Houlsby [19] and Abadie [21] showed that principles (1) and (2) could be empirically approximated by:

$$
\varepsilon_{p N}-\varepsilon_{p 0}=\Delta \varepsilon_{p}=T_{0}\left(\frac{\sigma_{p}}{\sigma_{0}}\right)^{m_{\sigma}} N^{m_{\alpha}}
$$

Equation 17

where $T_{0}$ is a dimensionless empirical factor equal to $0.5, m_{\sigma}$ is a load exponent equal to 4 , and $m_{\alpha}$ is equal to $0.31 . \sigma_{0}$ is a reference load, which can conveniently be chosen equal to the ultimate load and strength $k_{U}$ of the spring sliders (here equal to 1). Other published research studies in literature have recommended the use of a logarithmic law to capture accumulated deformation at peak load (e.g. [20, 30-32]). However, the use of a power laws significantly simplifies the following mathematical developments and was hence chosen here. However, it is noted that whether power law or logarithmic law descriptions are used, both account for an increase in deformation with cycle number in a reasonably similar manner.

The ratcheting behaviours (1) and (2) observed experimentally and summarized by Equation 17 can be captured in modelling through appropriate choice of the ratcheting rate function $\hat{R}$. The mathematical 
developments shown above and in Houlsby, Abadie [3], will not be altered by choosing a function instead of a constant, providing that $\hat{R}$ is a function of state $\left(\sigma, \varepsilon, \hat{\alpha}, \alpha_{r}\right.$ or $\left.\beta\right)$ and of the internal coordinate $\eta$. $\hat{R}$ can also be a function of $\hat{k}$, providing that $\hat{k}$ is itself a function of state and internal coordinate - which will be the case in this paper (see later Equation 22). The ratcheting rate can be a function of $\eta$, in which case the ratcheting rate of each surface is independent, or $\hat{R}$ can be the same for each surface $(\hat{R}(\eta) \equiv R)$. The paragraph below proposes a formulation for $\hat{R}$, adapted to capture principles (1) to (2).

\subsection{Decreased ratcheting rate with cyclic history}

First, the rate of ratcheting decreases with cyclic history. As the accumulated ratcheting strain $\beta$ encapsulates the ratcheting - or cyclic - history, this translates into choosing $\hat{R}$ to be a decreasing function of $\beta$. Equation 17 suggest the use of a decaying power-law:

$R_{n}=R_{o}\left(\frac{\beta}{\beta_{o}}\right)^{-m_{r}}$

Equation 18

$m_{r}$ is the exponent that defines the dependence of the ratcheting rate on the hardening parameter. If $m_{r}=0$, the rate of ratcheting remains constant (Figure $\left.1(b)\right) . \beta_{0}$ is the initial ratcheting strain, which is an arbitrarily small value and is introduced for normalisation purposes. Finally, $R_{0}$ is the initial rate of ratcheting. When $R_{0}=0$, ratcheting is disabled and the model produces a purely kinematic hardening response. Equation 18 enables the model to capture item (1).

\subsection{Increased ratcheting rate with load intensity}

An effective way of capturing the increased ratcheting rate with load magnitude is to make the ratcheting parameters $R_{n}$ a non-linear function of the load intensity $|\sigma|$. Similarly, Equation 17 suggests the use of a power-law:

$\hat{R}=R_{o}\left(\frac{\beta}{\beta_{o}}\right)^{-m_{r}}\left(\frac{|\sigma|}{\sigma_{0}}\right)^{m_{s}}$

Equation 19

The exponent $m_{s}$ defines the dependence of the ratcheting rate on the stress and enables the model to capture principle (2). 


\subsection{Independent rate within surfaces}

If described by Equation 19, the ratcheting rate is independent of the internal coordinate, and the rate of ratcheting induced by each surface is the same. However, there is a certain advantage in making the ratcheting rate caused by each surface proportional to the internal coordinate, and more specifically, to each of the surface strengths according to:

$$
\hat{R}=R_{o}\left(\frac{\hat{k}(\eta)}{k_{U}}\right)\left(\frac{\beta}{\beta_{o}}\right)^{-m_{r}}\left(\frac{|\sigma|}{\sigma_{0}}\right)^{m_{s}}
$$

As $\hat{k}(\eta)$ is related to the stress level, this partly satisfies principle (2), while making the ratcheting rate grow in proportion to the strength of that surface.

Moreover, when substituting Equation 20 in Equation 9 (or Equation 11), the internal variable for ratcheting now becomes a function of the plastic work. Ignoring ratcheting for the time being $\left(R_{0} \equiv 0\right)$, and considering a purely kinematic hardening response for 1-directional loading, the series and parallel models, as described in Houlsby, Abadie [3] have a different definition of plastic strain, but can produce exactly the same stress-strain response (through appropriate choice of $\widehat{k}$ and $\widehat{H}$ [24]). Consequently, the work done at any step within the loop is the same in both models. By expressing the ratcheting strain as a function of the plastic work instead of the plastic strain, the p-HARM and s-HARM models become indistinguishable for uniaxial loading. This option offers the opportunity of considering both strain and stress-controlled scenarios, while using both models for numerical efficiency, with the confidence that the results obtained will be identical. In addition, while the kinematic hardening parameters $(\hat{k}, \widehat{H})$ are different because their definition in each model is fundamentally different, the ratcheting parameters, on the other hand, $\left(R_{0}, \beta_{0}, m_{r}, m_{s}\right)$ have the same definition and hence, the same values. Therefore, the results of the mathematical developments shown below for the s-HARM model, also apply to the p-HARM model for this particular expression of the ratcheting rate.

For implementation purposes, the ratcheting rate may be expressed in its discrete form following: 
$R_{n}=R_{o}\left(\frac{k_{n}}{k_{U}}\right)\left(\frac{\beta}{\beta_{o}}\right)^{-m_{r}}\left(\frac{|\sigma|}{\sigma_{0}}\right)^{m_{s}}$

Equation 21

Equation 20 (or Equation 21) implements principles (1) and (2) in a reasonable and efficient way. Principle

(3) has been left aside for the time being and will be addressed in Section 5.3.

\subsection{List of parameters to calibrate}

The above framework requires the calibration of nine parameters summarized in Table 2 . Some of these parameters are very straightforward $\left(E_{0}, k_{U}, \epsilon_{p u}\right)$ to determine. Importantly, all the parameters have a physical meaning and can be derived analytically, using the fit from a monotonic test (Equation 16) and the fit of the evolution of accumulated deformation with cycle number from a few long-term continuous cyclic tests (Equation 17), following the methodology described below.

Table 2. List of parameters for calibration of HARM model: definition, analytical expressions and values

\begin{tabular}{|c|c|c|c|c|c|}
\hline & Definition & Calibration method & \multicolumn{2}{|c|}{ Value used in paper } & Eq. No. \\
\hline \multicolumn{3}{|c|}{ Backbone curve (kinematic hardening) } & Analytical & Optimised & \\
\hline$E_{0}$ & Initial stiffness & Tangent to origin of backbone & \multicolumn{2}{|c|}{59} & - \\
\hline $\boldsymbol{k}_{n}$ & Kinematic hardening strengths & $k_{n}=k_{U} \frac{n}{N_{S}}$ & \multicolumn{2}{|c|}{$N_{S}=40$} & $\begin{array}{c}\text { Equation } \\
22\end{array}$ \\
\hline $\boldsymbol{H}_{\boldsymbol{n}}$ & Kinematic hardening stiffnesses & $\hat{H}(\eta)=\frac{N_{S}}{m_{h}\left(m_{h}-1\right)} \frac{k_{U}}{\varepsilon_{p U}}\left(\frac{\eta}{N_{S}}\right)^{2-m_{h}}$ & $m_{h}=3$ & $\begin{array}{c}H_{n} \text { values } \\
\text { recomputed }\end{array}$ & $\begin{array}{c}\text { Equation } \\
24\end{array}$ \\
\hline $\boldsymbol{k}_{U}$ & Ultimate strength & Ultimate limit load of the pile & & $L$ & - \\
\hline$\varepsilon_{p u}$ & ultimate monotonic plastic strain & Strain at ultimate load & & 1 & - \\
\hline \multicolumn{6}{|c|}{ Ratcheting behaviour } \\
\hline$\beta_{0}$ & Initial ratcheting strain & Arbitrarily small value & \multicolumn{2}{|c|}{$1 e-4$} & - \\
\hline$m_{r}$ & $\begin{array}{l}\text { Exponent defining the decrease } \\
\text { of ratcheting rate with cyclic } \\
\text { history }\end{array}$ & $m_{r}=\frac{1}{m_{\alpha}}-1$ & 2.2 & 3.1 & $\begin{array}{c}\text { Equation } \\
30\end{array}$ \\
\hline $\boldsymbol{m}_{\mathrm{s}}$ & $\begin{array}{l}\text { Exponent defining the increase of } \\
\text { ratcheting rate with load } \\
\text { intensity }\end{array}$ & $m_{s}=m_{\sigma}\left(m_{r}+1\right)-m_{h}-1$ & 8.9 & 9.5 & $\begin{array}{c}\text { Equation } \\
31\end{array}$ \\
\hline $\boldsymbol{R}_{\mathbf{0}}$ & Initial ratcheting rate & $R_{\beta}=R_{0} \beta_{0}^{m_{r}}=\frac{T_{0}^{m_{r}+1}}{\mathcal{E}_{p U} \kappa_{m 0} \kappa_{m}}$ & 1.7 & 1.8 & $\begin{array}{c}\text { Equation } \\
32\end{array}$ \\
\hline
\end{tabular}

\section{Closed form solution for calibration}

\subsection{Expression of plastic strain on yielding}

First, we assume a specific form of the distribution of yield surfaces, the expression for $\hat{k}$ is simply: 
$\hat{k}(\eta)=k_{U} \frac{\eta}{N_{S}}$

Equation 22

According to Puzrin and Houlsby [25], the expression for $\widehat{H}(\eta)$ is then derived from:

$\hat{H}\left(\sigma N_{S} / k_{U}\right)=\frac{N_{S}}{k_{U}}\left(\frac{d^{2} \varepsilon}{d \sigma^{2}}\right)^{-1}$

Equation 23

Substituting Equation 16 into Equation 23 and Equation 9, this gives:

$\hat{H}(\eta)=\frac{N_{S}}{m_{h}\left(m_{h}-1\right)} \frac{k_{U}}{\varepsilon_{p U}}\left(\frac{\eta}{N_{S}}\right)^{2-m_{h}}$

Equation 24

Equation 20 and Equation 22 are used to calibrate the backbone curve.

\subsection{Ratcheting strain}

Calibration of the accumulation of ratcheting strain with cycle number is achieved using:

$\beta_{p N}^{m_{r}+1}=\beta_{0}^{m_{r}+1}+\varepsilon_{p U} R_{\beta} \kappa_{m 0}\left(1+\kappa_{m} N\right)\left(\frac{\sigma_{p}}{k_{U}}\right)^{m_{s}+m_{h}+1}$

Equation 25

with:

$R_{\beta}=R_{0} \beta_{0}^{m_{r}}$

Equation 26

$\kappa_{m 0}=\frac{\left(m_{h}-1\right)\left(m_{r}+1\right)}{m_{s}+m_{h}+1}$

Equation 27

$\kappa_{m}=\frac{1}{2^{m_{h}}}\left(\left(m_{s}+m_{h}+1\right) \mathrm{B}\left(m_{s}+1, m_{h}+1\right)+1\right)$

Equation 28

where $B(.,$.$) is the Beta function. Demonstration of Equation 25$ is provided in Appendix A. As the $R_{0}$ value is proportional to $\beta_{0}{ }^{-m_{r}}$, with $\beta_{0}$ being a very small value, the value of $R_{0}$ can rapidly become a very large number, while the ratcheting rate $\hat{R}$ still remains a small fraction. It is hence sensible to introduce $R_{\beta}$ (Equation 26), which is invariant to the choice of $\beta_{0}$ value and represents more closely the value of the initial rate of ratcheting to calibrate for design. 


\section{Application to prediction of test results}

\subsection{Analytical expression for parameters}

For the test results used here, the values of $R_{\beta}, m_{r}$ and $m_{s}$ are typically in the range of $1,2.5$ and 8.5 respectively, and in this paper, $\beta_{0}$ was chosen equal to $\beta_{0}=10^{-4} \times \varepsilon_{p U}$, though any small quantity would provide the same results. In the worst case of a large cyclic load of $1, \beta_{0}{ }^{m_{r}+1} \approx 10^{-14}$, $\varepsilon_{p U} R_{\beta} \kappa_{m 0}\left(\frac{\sigma_{p}}{k_{U}}\right)^{m_{s}+m_{h}+1} \approx 0.6$ and $\beta_{p U} R_{\beta} \kappa_{m 0} \kappa_{m}\left(\frac{\sigma_{p}}{k_{U}}\right)^{m_{s}+m_{h}+1} \approx 0.07$. Hence, for cycle number 1000 and above: $\beta_{0}^{m_{r}+1} \ll \varepsilon_{p U} R_{\beta} \kappa_{m 0}\left(\frac{\sigma_{p}}{k_{U}}\right)^{m_{s}+m_{h}+1} \ll \varepsilon_{p U} R_{\beta} \kappa_{m 0} \kappa_{m} N\left(\frac{\sigma_{p}}{k_{U}}\right)^{m_{s}+m_{h}+1}$, which simplifies Equation 25 . Equally, it is reasonable to assume that, at large cycle number, the amount of ratcheting strain accumulated during cyclic loading becomes sufficiently large that the accumulated ratcheting strain caused during the initial load is negligible, and therefore: $\beta_{p N}-\beta_{p 0} \approx \beta_{p N}$. Accordingly, at large cycle number, Equation 25 gives:

$\beta_{p N}-\beta_{p 0} \approx\left(\varepsilon_{p U} R_{\beta} \kappa_{m 0} \kappa_{m} N\left(\frac{\sigma_{p}}{k_{U}}\right)^{m_{s}+m_{h}+1}\right)^{\frac{1}{m_{r}+1}}$ Equation 29

Identifying Equation 29 with Equation 15 and Equation 17 yields to:

$m_{r}=\frac{1}{m_{\alpha}}-1$

Equation 30

$m_{s}=m_{\sigma}\left(m_{r}+1\right)-m_{h}-1$

Equation 31

$R_{\beta}=\frac{T_{0}^{m_{r}+1}}{\mathcal{E}_{p U} \kappa_{m 0} \kappa_{m}}$

Equation 32

With the value of $m_{\alpha}, m_{\sigma}$ and $T_{0}$ provided in Section 2.3 , this gives: $R_{\beta}=1.7, m_{r}=2.2$ and $m_{s}=8.9$. The expression and value used for the calibration of parameters is summarized in Table 2. For implementation purposes, the $k_{n}$ and $H_{n}$ have been expressed in their discrete form. 


\subsection{Correction of the backbone curve}

Adding ratcheting to the kinematic hardening model leads to a shift of the backbone curve due to the additional accumulated ratcheting deformation during initial loading, which is not accounted for in the calibration of $\hat{k}$ and $\widehat{H}$ in Equation 22, Equation 23 and Equation 24. This is demonstrated in Figure 4, where the backbone curve obtained through pure kinematic hardening $\left(R_{0} \equiv \hat{R} \equiv 0\right)$ is compared with that once ratcheting is added, both of which are compared with the data of the monotonic test (MCo). To adjust for this effect, a corrective sub-routine can be added to balance the additional ratcheting strain by stiffening the backbone curve so as to precisely capture the measurements. This is also shown in Figure 4, where the values of $\widehat{H}$ were optimised until the predicted backbone curve matched the test data.

\subsection{Final modelling}

An example of the prediction of the three continuous cyclic test results CMLT1, 2 and 3 using the above calibration procedure, without the correction of the backbone curve is shown in Figure 5(a). The graph displays the strain at minimum peak load, also called residual deformation following cyclic loading, which is of great interest for design. Comparison with experimental data proves that the mathematical developments leading to Equation 30, Equation 31 and Equation 32 provide accurate prediction of the trend and magnitude observed.

Figure 5(a) displays the results computed incrementally, cycle by cycle, and those obtained using the accelerated form (Equation 11), with the acceleration programme used by Abadie, Byrne [29, Table 3] . The graph demonstrates that the use of a non-linear expression for the ratcheting rate (Equation 21) provides an exact result when used in its accelerated form. 


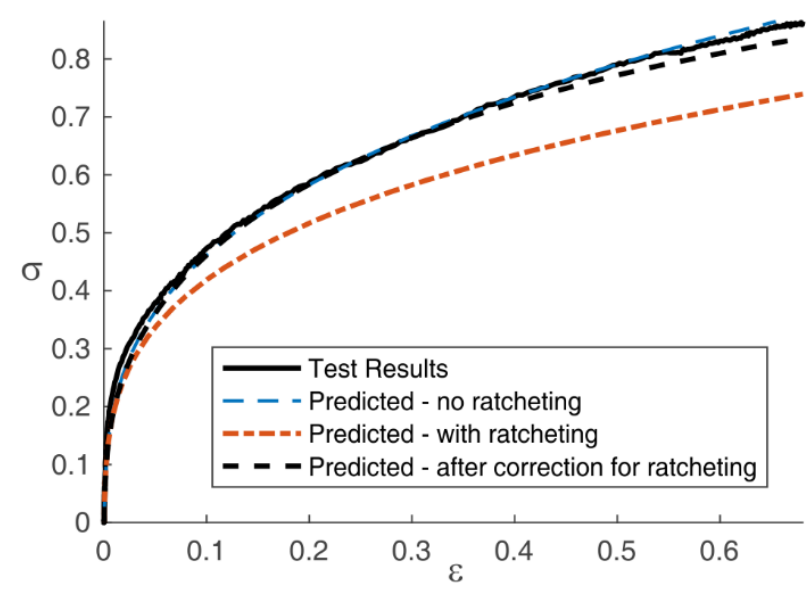

Figure 4. Prediction of the backbone curve (test $M C o)$ without ratcheting $\left(R_{\beta}=0\right)$ and with ratcheting $\left(m_{r}=2.2, m_{s}=8.9\right.$, $\left.R_{\beta}=1.7\right)$, with and without optimisation correction of the $H_{n}$

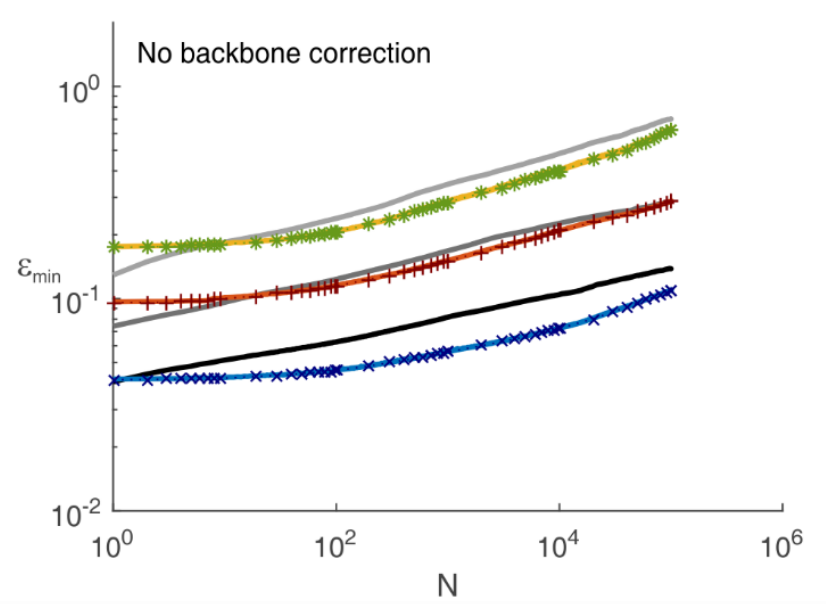

(a)

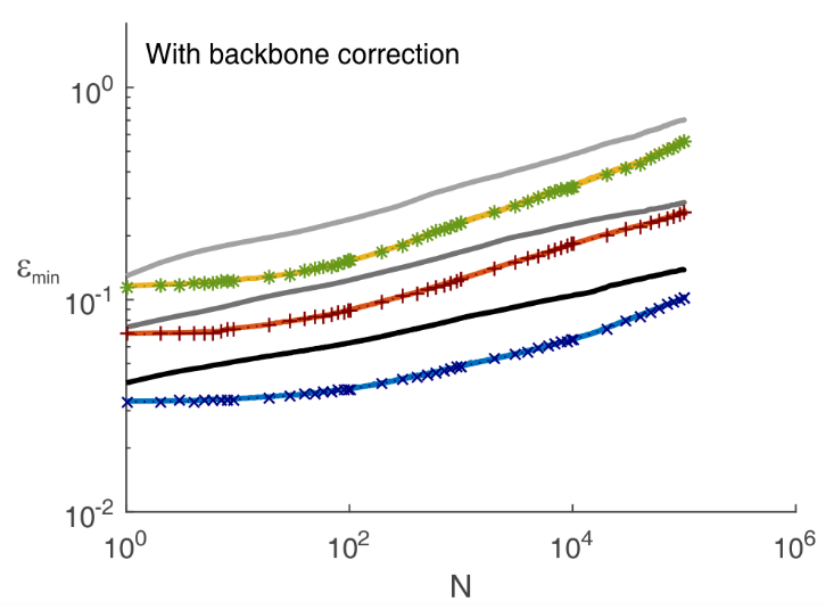

(b)
Test CMLT1

Test CMLT2

Test CMLT3
HARM Incremental CMLT1

HARM Incremental CMLT2

HARM Incremental CMLT3
... HARM Accelerated CMLT1

... HARM Accelerated CMLT2

*... HARM Accelerated CMLT3

Figure 5. Prediction of the residual deformation at minimum peak load for tests CMLT1,2 and 3 with parameters provided by Equation $30\left(m_{r}=2.2\right)$., Equation $31\left(m_{s}=8.9\right)$ and Equation $32\left(R_{\beta}=1.7\right)$, (a) prior and (b) post-optimisation of the $\mathrm{Hn}$ for correction of the backbone curve (Figure 4)

However, accurate prediction of the backbone curve is crucial to any subsequent attempt in predicting the response to multi-amplitude cyclic loading. This is because any load exceeding maximum past cyclic load will induce the response to follow that of the backbone curve (Masing behaviour). When adding the correction of the $\widehat{H}$ aforementioned in Section 4.2 (black dotted line in Figure 4), the response then obtained is that displayed in Figure 5(b). Clearly, the modification of $\widehat{H}$ changes the mathematical 
developments of Appendix A, and in particular, Equation 43, which explains the loss in accuracy observed in Figure 5(b).

At this stage, there are two options:

(i) Use the prediction with no correction of the backbone, with the understanding that any prediction in capacity and long-term displacement will be conservative (Figure 5(a)) or

(ii) Use Equation 30, Equation 31 and Equation 32 with optimised values of $\widehat{H}$ (Figure $5(\mathrm{~b})$ ) as initial solution for further optimisation of parameters $R_{\beta}, m_{r}$ and $m_{s}$

Although the second option may seem the obvious choice, it provides a less satisfactory fit for 2-way loading, which is not addressed in this paper. Option (ii) is hence pursued in the following and using the results of tests CMLT1,2 and 3, an iterative procedure is used to recompute the values of $R_{\beta}, m_{r}$ and $m_{s}$. This process is made more efficient by using the accelerated form of the model. For each new set of $R_{\beta}$, $m_{r}$ and $m_{s}$ values tested, the values of $\widehat{H}$ were re-computed to enable close match of the backbone curve (similar to that of Figure 4) until a close prediction of test MCo and CMLT1,2,3 were obtained. This provided the optimised valules $R_{\beta}=1.8, m_{r}=3.1$ and $m_{s}=9.5$. Figure 6 shows the experimental test results compared with the HARM prediction for residual deformation and accumulated deformation at maximum peak load (Equation 15), demonstrating high accuracy in the prediction for large cycle numbers. This is in agreement with the calibration choice of section 4.1. Most importantly, Figure 6(c) and (d) displays the compared load-deflection curves for the three tests, showing that the load-deflection curves can be predicted with remarkable accuracy for use in structure design. 


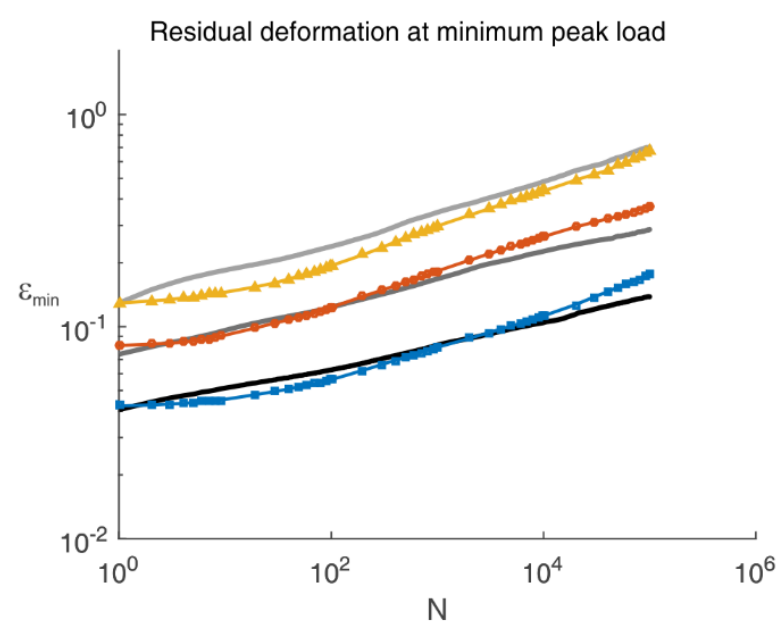

(a)

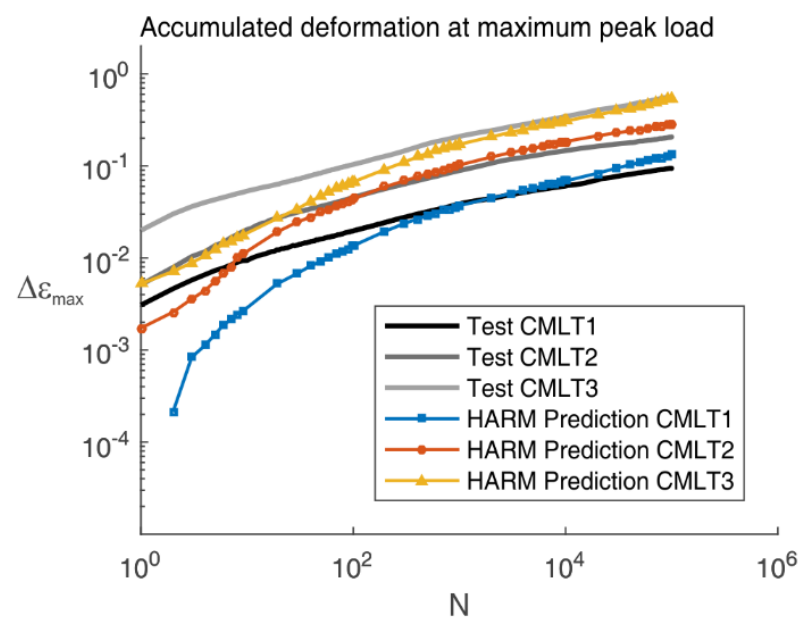

(b)

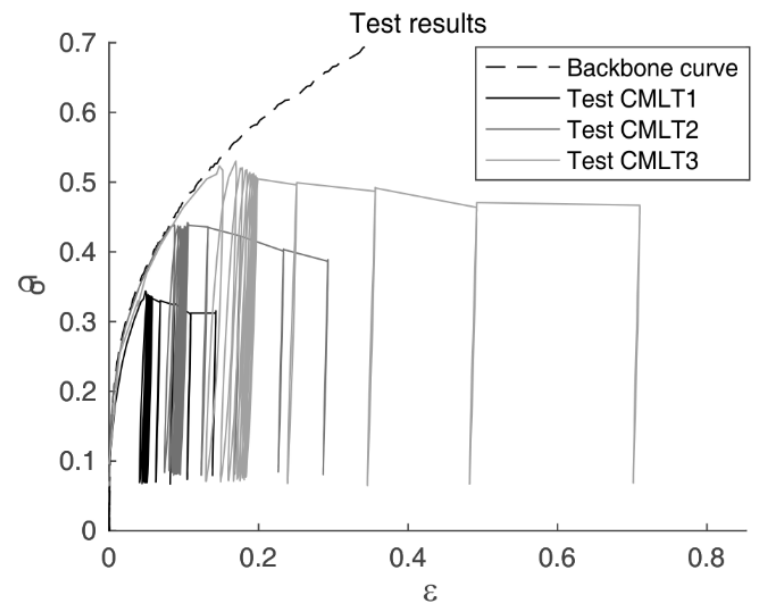

(c)

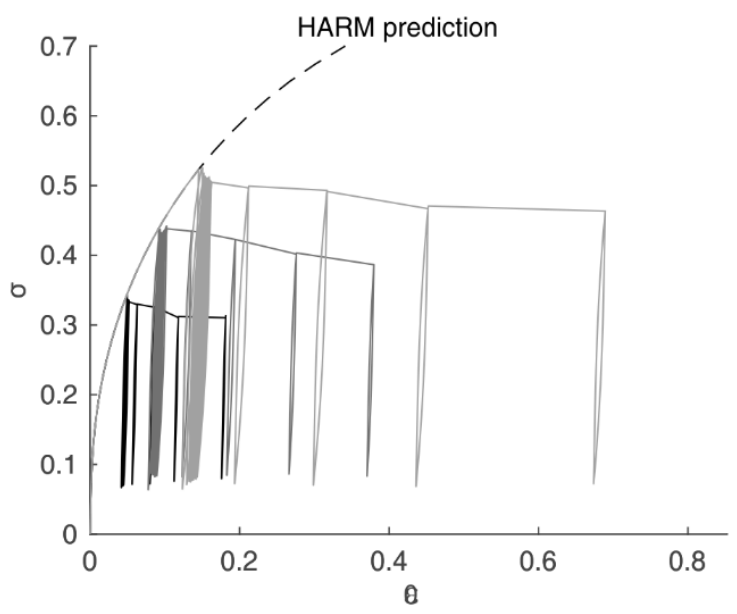

(d)

Figure 6. Final prediction of (a) the residual deformation at minimum peak load, (b) the accumulated deformation at maximum peak load and (d) the load-deflection curves for $(c)$ tests results CMLT1,2 and $3\left(m_{r}=3.1, m_{s}=9.5, R_{\beta}=1.8\right)$

\subsection{Evolution of ultimate capacity}

The above feature therefore enables prediction of ultimate capacity following long-term cyclic loading, also called monotonic re-loading. For tests CMLT1,2 and 3, this is shown in Figure 7, where the monotonic re-loading data from Abadie, Byrne [1] are reported and compared with the calibrated HARM response, showing good agreement. For clarity of the chart, the past cyclic loading history is discarded here and solely shown through the initial point of re-load, corresponding to the residual strain following cyclic loading. 
Similarly, Figure 8 shows the compared response of monotonic re-loading following cyclic loading at maximum load $\zeta_{b}=0.42$ for a range of cycle numbers (Tests CMC1-5 + CMLT2, Table 1 ), also demonstrating good agreement.

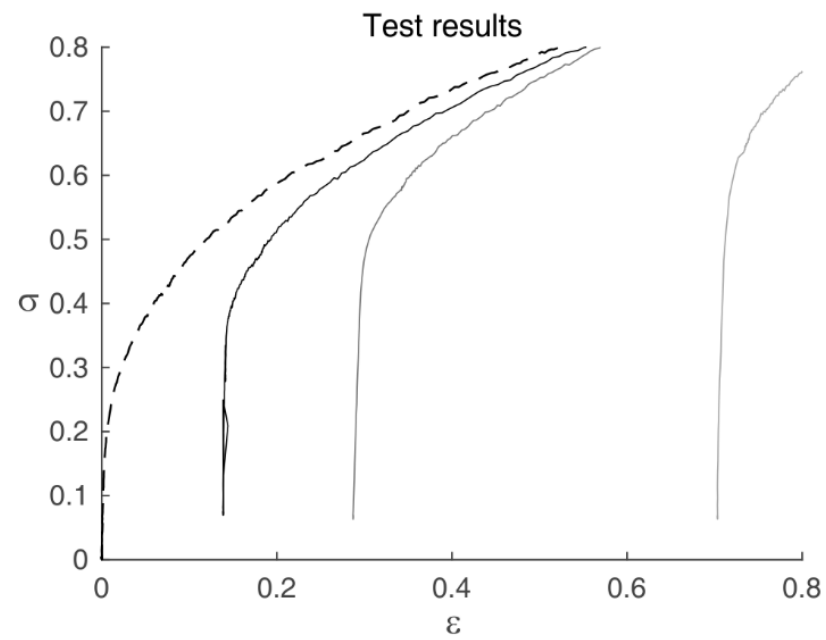

(a)

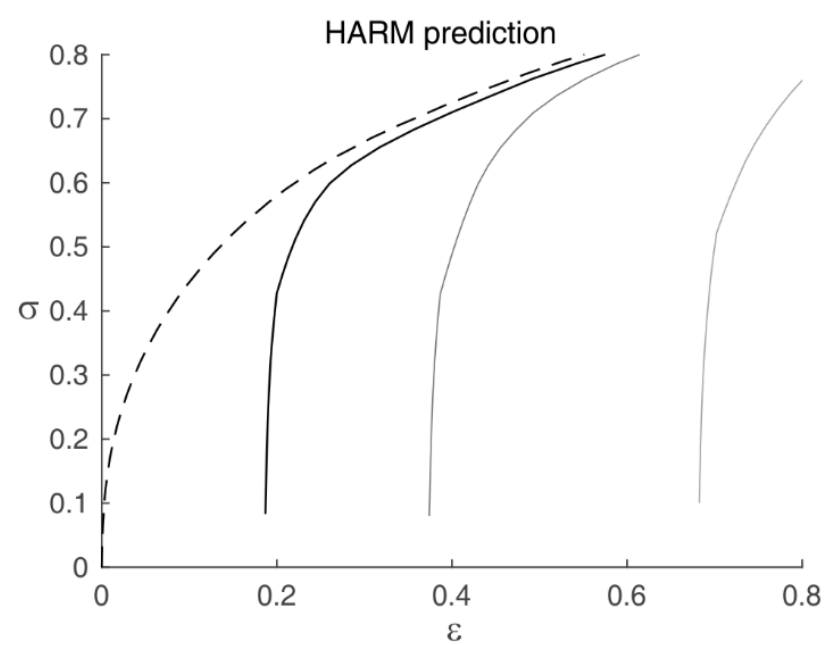

(b)

$\begin{array}{lr}---\mathrm{CMCO} & \mathrm{CMLT2}-\zeta_{\mathrm{b}}=0.42 \\ -\mathrm{CMLT1}-\zeta_{\mathrm{b}}=0.31 & \mathrm{CMLT3}-\zeta_{\mathrm{b}}=0.47\end{array}$

Figure 7. Compared (a) experimental data and (b) HARM prediction for monotonic reload following long-term continuous cyclic tests CMLT1,2 and 3

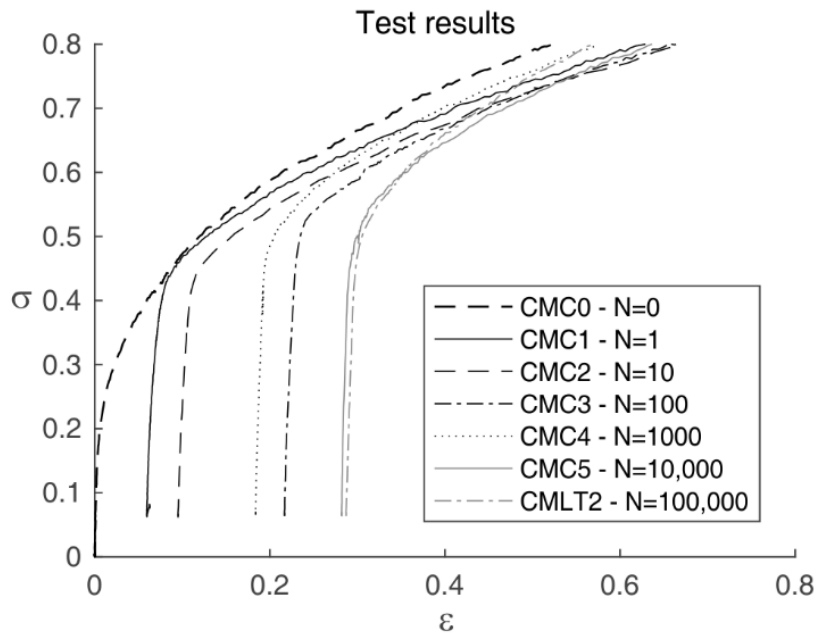

(a)

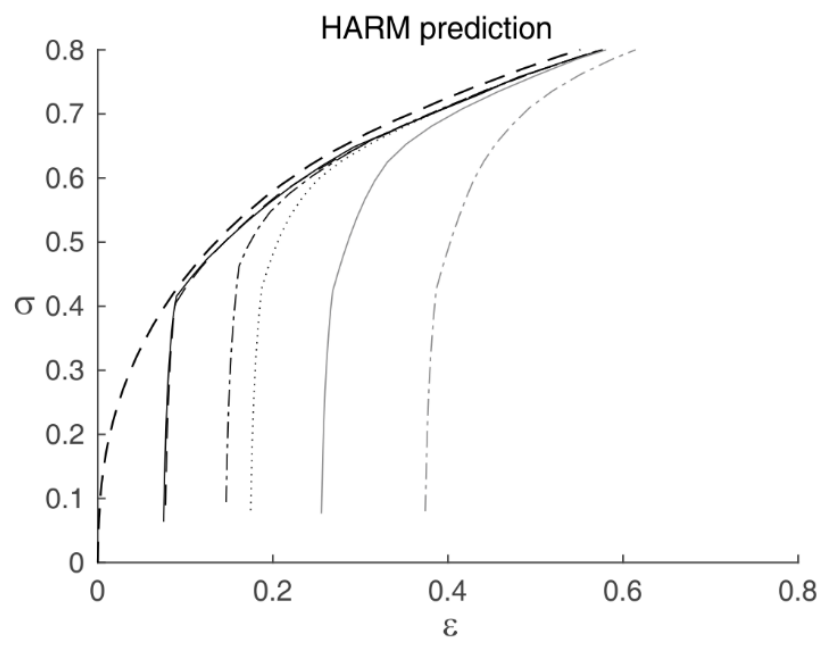

(b)

Figure 8. Compared (a) experimental data and (b) HARM prediction for monotonic reload following long-term continuous cyclic tests at load magnitude $\mathbf{0 . 4 2}$ for a range of cycle number

\subsection{Multi-amplitude cyclic loading}

Figure 9 shows the compared response for a simple 1-way cyclic loading test of 1 cycle at increasing load magnitude (Test H1IM, Table 1), demonstrating that the Masing behaviour is the primary effect captured and that accurate prediction of the backbone curve enables close match of the cyclic responses. 
The above provides confidence that the calibrated HARM framework can be used to predict multiamplitude load scenarios and this is shown through prediction of selected multi-amplitude tests (Table 1):

- A series of 3 large load events in different order, tests MALL1,2,3 from Abadie, Byrne [1]

- A series of 3 combined long-term small and short-term large load events in different order, tests MASL1,2,3 from Abadie, Byrne [1] and

- Two more complex load scenarios extracted from Abadie, Byrne [2], tests MA1 and 2 [and equivalent to MASL6 and 7 in 21].

The results are shown in Figure 10, Figure 11 and Figure 12 respectively. They demonstrate that the calibrated HARM prediction captures the observed behaviour fairly well, except for cases MALL2 and MASL3, where a very large load is first applied and then followed by cyclic load packets at lower magnitude. In these case, the accumulated ratcheting strain generated by the cyclic load is underestimated. This is caused by accumulation of hardening during initial loading, which, because of the term $\left(\frac{\beta}{\beta_{0}}\right)^{-m_{r}}$, slows down the ratcheting rate too much. Further work on this would be required in order to obtain a good balance between hardening and ratcheting. 


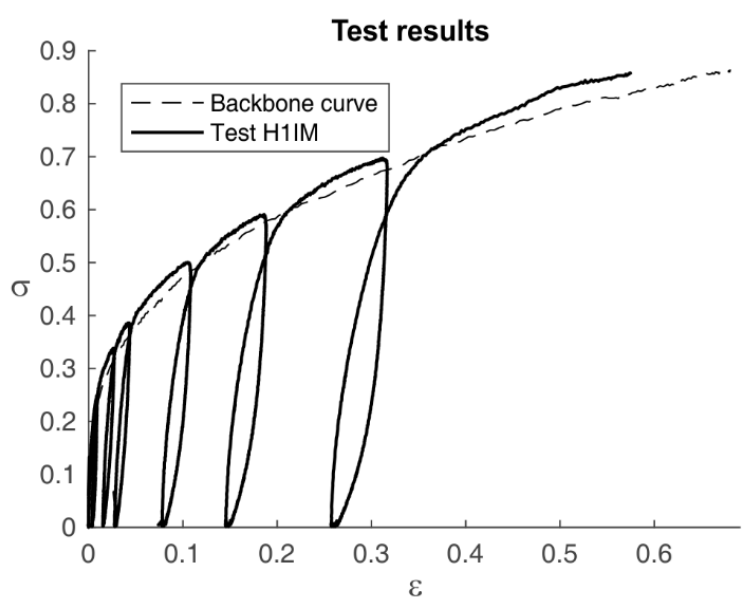

(a)

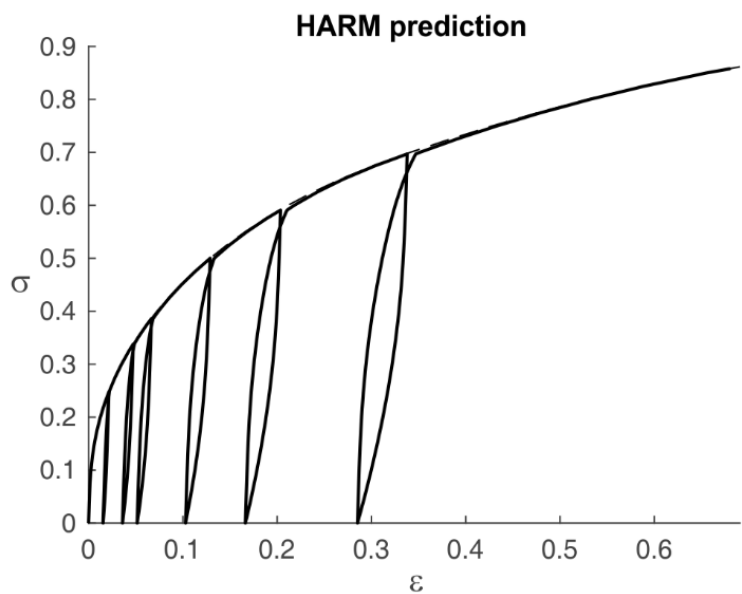

(b)

Figure 9. Compared (a) experimental data and (b) HARM prediction for test H1IM 


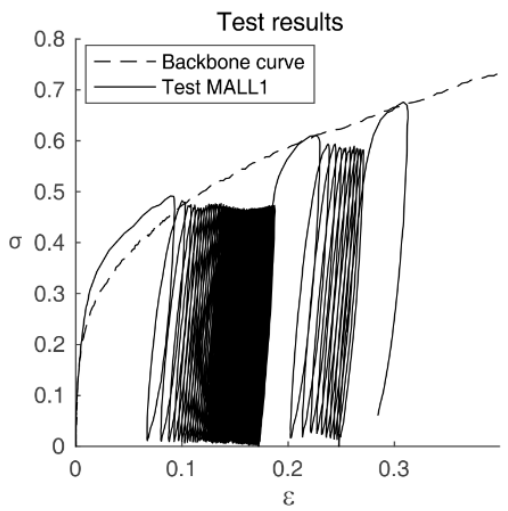

(a)

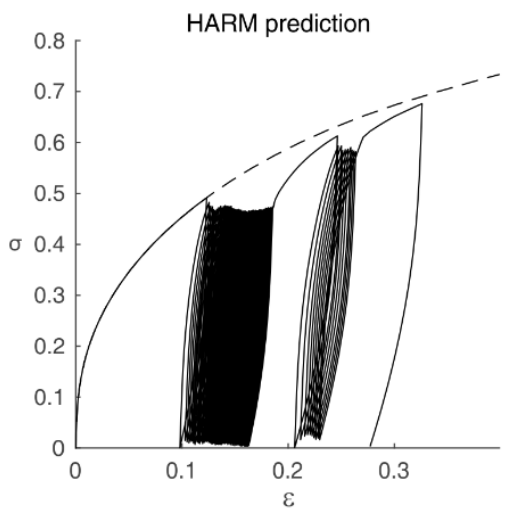

(d)

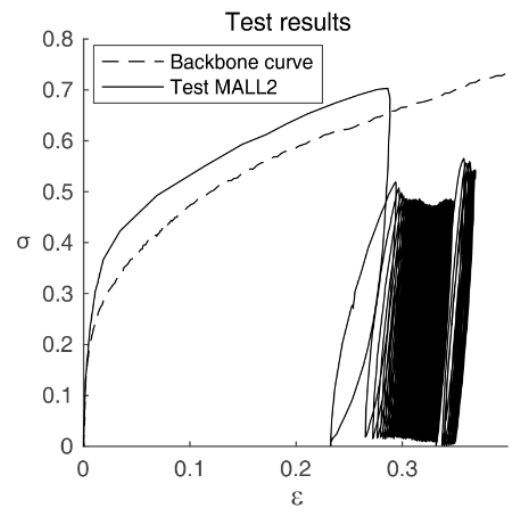

(b)

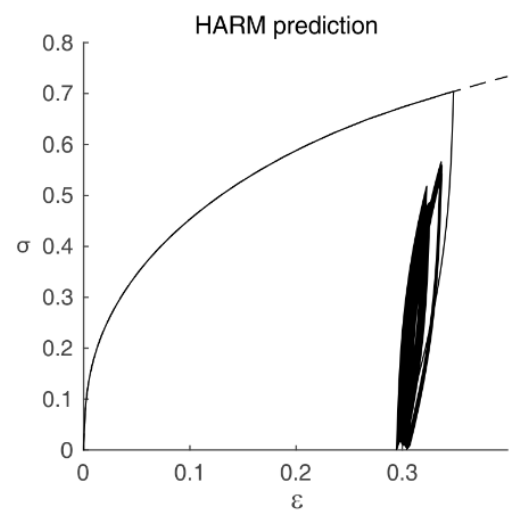

(e)

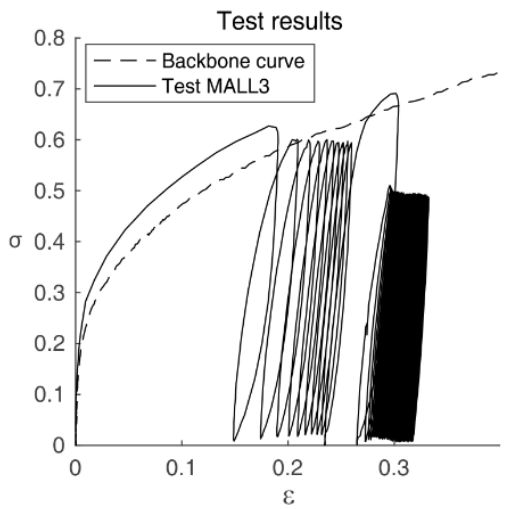

(c)

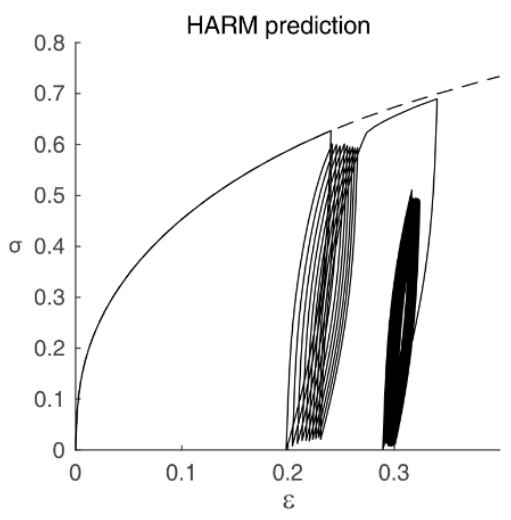

(f)

Figure 10. Compared $(a, b, c)$ experimental data and (d,e,f) HARM prediction for test MALL1,2,3

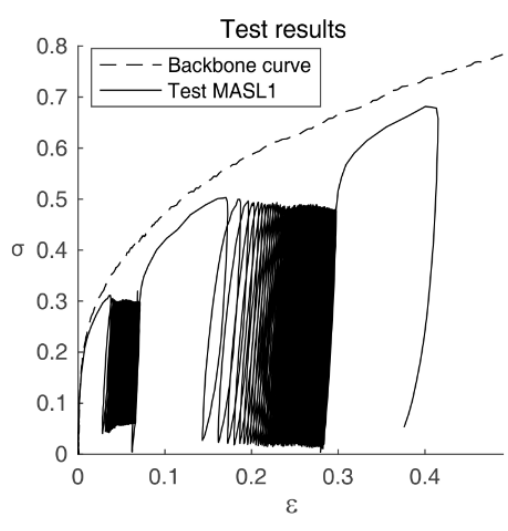

(a)

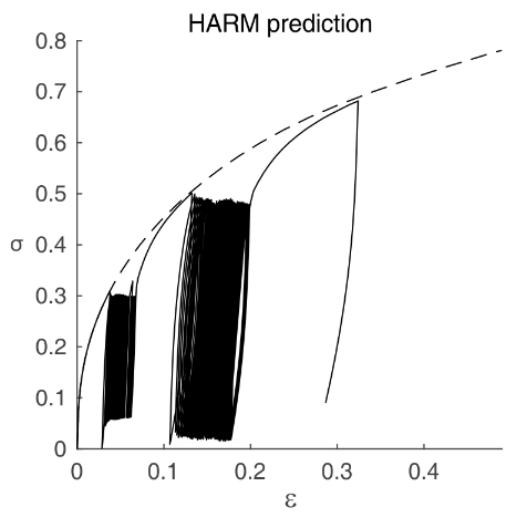

(d)

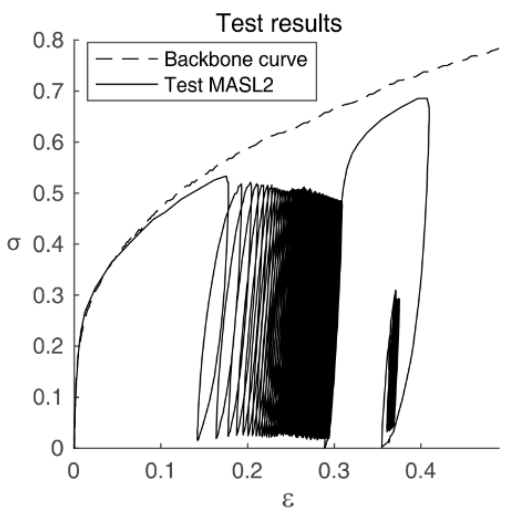

(b)

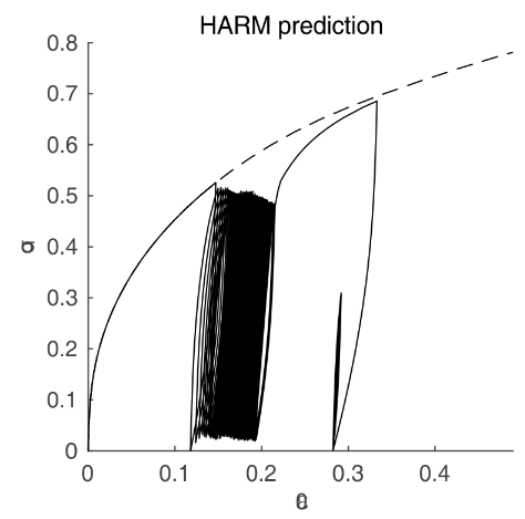

(e)

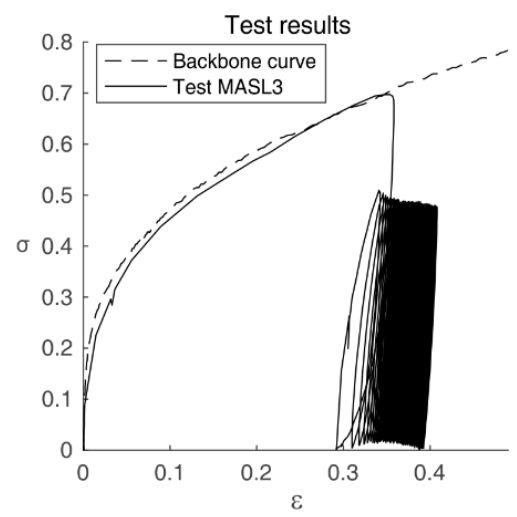

c)

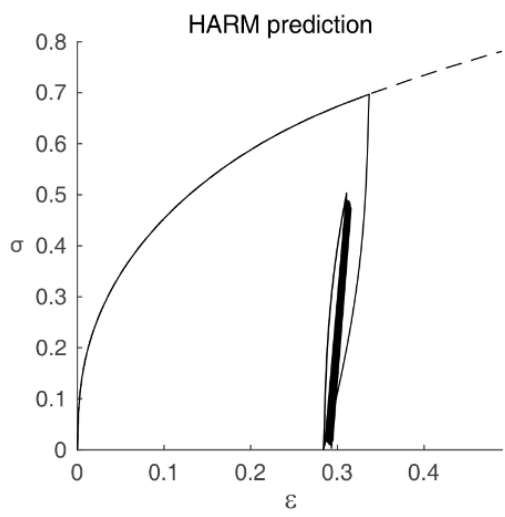

(f)

Figure 11. Compared $(a, b, c)$ experimental data and (d,e,f) HARM prediction for test MASL1,2,3 


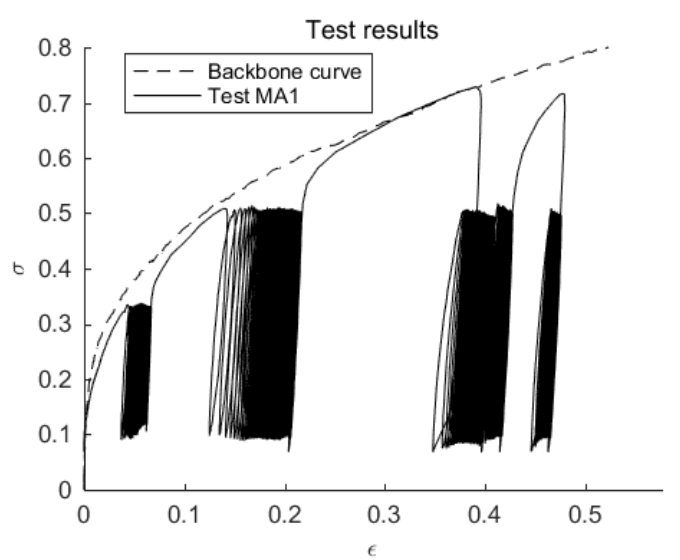

(a)

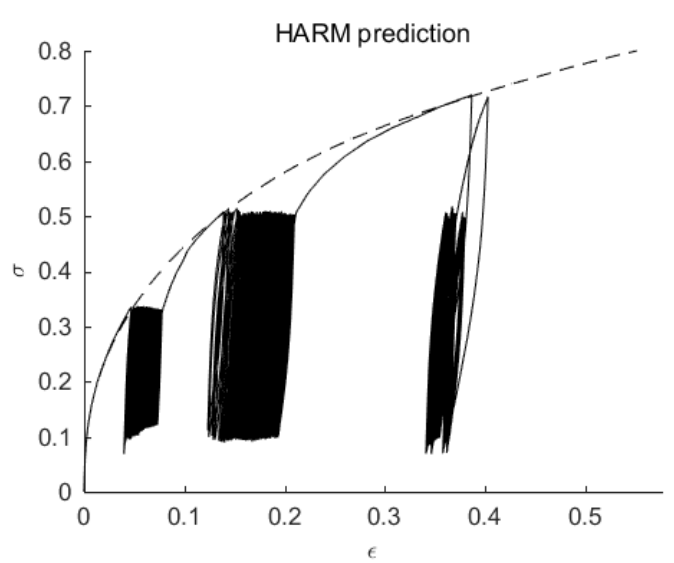

(c)

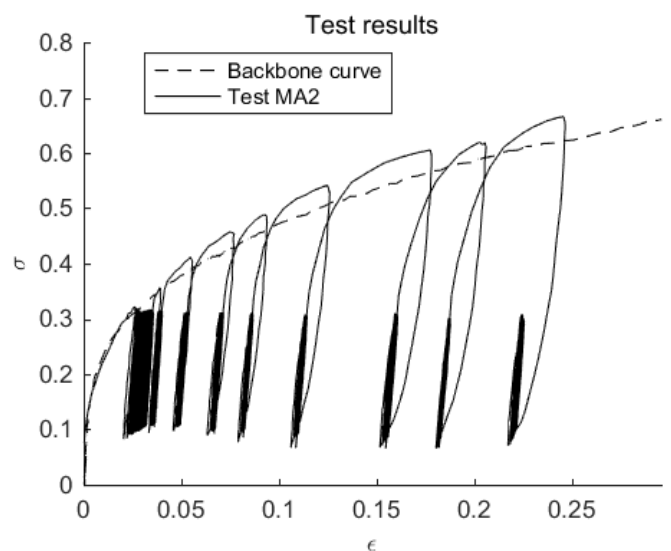

(b)

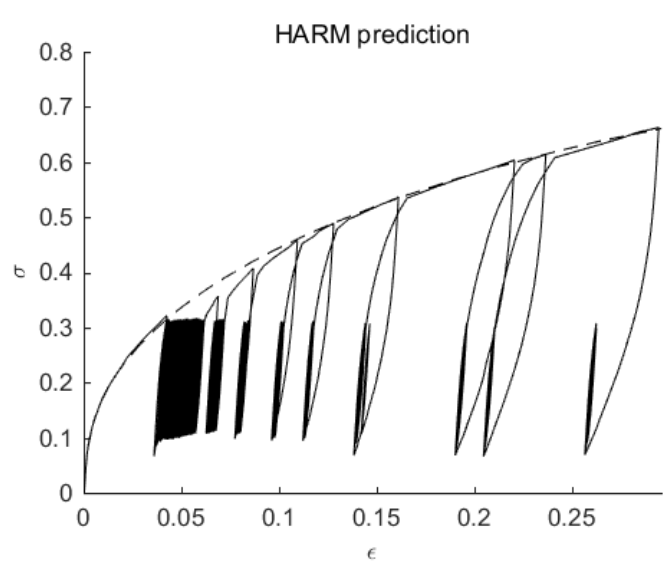

(d)

Figure 12. Compared $(a, b)$ experimental data and $(c, d)$ HARM prediction for test MA1,2

\section{Discussion}

\subsection{Different ratcheting rate form}

The methodology described above can be applied and derived for a wide range of ratcheting rate forms, providing that $\hat{R} \hat{\alpha}$ is integrable on $\mathbb{R}^{+}$(Appendix A, Equation 41), which is facilitated by the choice of power-laws for the backbone curve fit and the ratcheting rate expression $\hat{R}$. In particular, if one prefers to use Equation 19 instead of Equation 20 for $\hat{R}$, Equation 25 then becomes (see Appendix A.4 for demonstration):

$\beta_{p N}^{m_{r}+1}=\beta_{0}^{m_{r}+1}+\varepsilon_{p U} R_{\beta} \kappa_{m 0}^{*}\left(1+\kappa_{m}^{*} N\right)\left(\frac{\sigma_{p}}{k_{U}}\right)^{m_{S}+m_{h}}$

with: 
$\kappa_{m 0}^{*}=\frac{m_{h}\left(m_{r}+1\right)}{m_{s}+m_{h}}$

Equation 34

$\kappa_{m}^{*}=\frac{1}{2^{m_{h}-1}}\left(\left(m_{s}+m_{h}\right) \mathrm{B}\left(m_{s}+1, m_{h}\right)+1\right)$

Equation 35

Applying the same reasoning as in Section 4.1, the analytical expressions for Equation 30, Equation 31 and Equation 32 are:

$m_{r}=\frac{1}{m_{\alpha}}-1$

Equation 36

$m_{s}=m_{\sigma}\left(m_{r}+1\right)-m_{h}$

Equation 37

$R_{\beta}=\frac{T_{0}^{m_{r}+1}}{\mathcal{E}_{p U} \kappa_{m 0}^{*} \kappa_{m}^{*}}$

Equation 38

These equations give: $R_{\beta}=0.56, m_{r}=2.2$ and $m_{s}=9.9$ and provide the comparable results, however, equivalence between s-HARM and p-HARM is then lost.

\subsection{Elasto-plastic threshold}

The above equations were derived assuming a non-linear plastic behaviour, with no elastic region and an elastic-plastic threshold equal to zero, which is well suited to the case studied here. It is possible however, to consider a non-zero threshold, which makes the mathematical developments slightly more complex. A framework for the introduction of such threshold is shown in Abadie [21].

\subsection{Hardening and hysteresis loop shape evolution: prediction of stiffness and damping}

The above framework has not specifically addressed principle (3). In fact, because the model obeys Masing rules and the change in ratcheting rate does not affect the hysteresis loop shape, both the secant stiffness and loop area remain constant during long-term cyclic loading at constant load magnitude. However, for tests CMLT1,2 and 3, the load magnitude drops slightly over time, and may induce some of the changes in secant stiffness and loop area observed by Abadie, Byrne [1]. When modelling tests CMLT1,2,3 using the calibrated HARM model (Figure 6), the change in load magnitude was accounted for and it was therefore possible to compute the evolution of stiffness and area as obtained with the current version of 
the model. The results are displayed in Figure 13 and show that (i) the change in load magnitude is not sufficient to explain the change in hysteresis loop area, with further stiffening occurring as outlined by LeBlanc, Houlsby [19] and Abadie, Byrne [1], and (ii) current modelling is not sufficient to capture the trend accurately. Further hardening of the response with cycle number needs to be introduced through mechanisms similar to isotropic hardening, and this is also obvious in test MA2 (Figure 12(b)).

An efficient option for capturing the change in hysteresis loop shape is to modify the surface strengths $\hat{k}$ with cyclic history, as this does not change the mathematical developments shown in Houlsby, Abadie [3]. A natural way to achieve this is to choose an increasing function of $\beta$, which gradually stiffens the overall response on each unload-reload loop and both tightens the hysteresis loop shape and increases the secant stiffness. Using a power-law, for example, one can choose:

$\hat{k}(\eta)=\hat{k}_{0}(\eta) \cdot\left(\frac{\beta}{\beta_{o}}\right)^{m_{k}}$

Equation 39

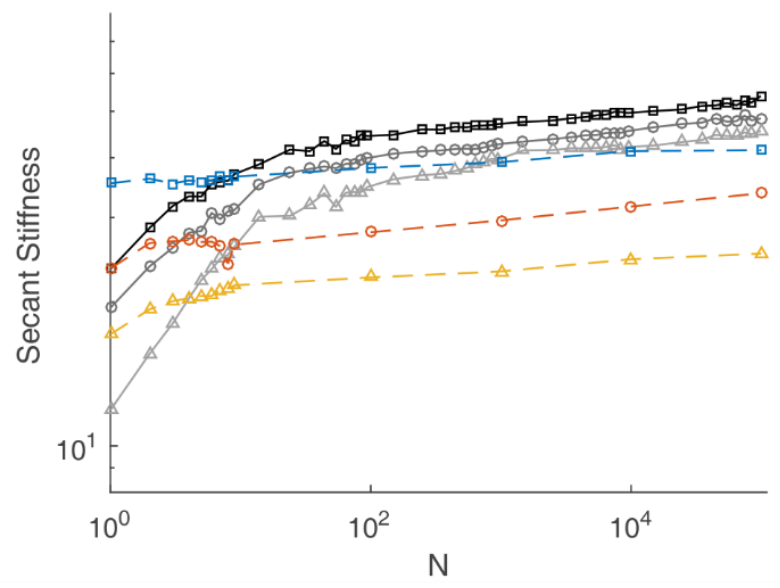

(a)

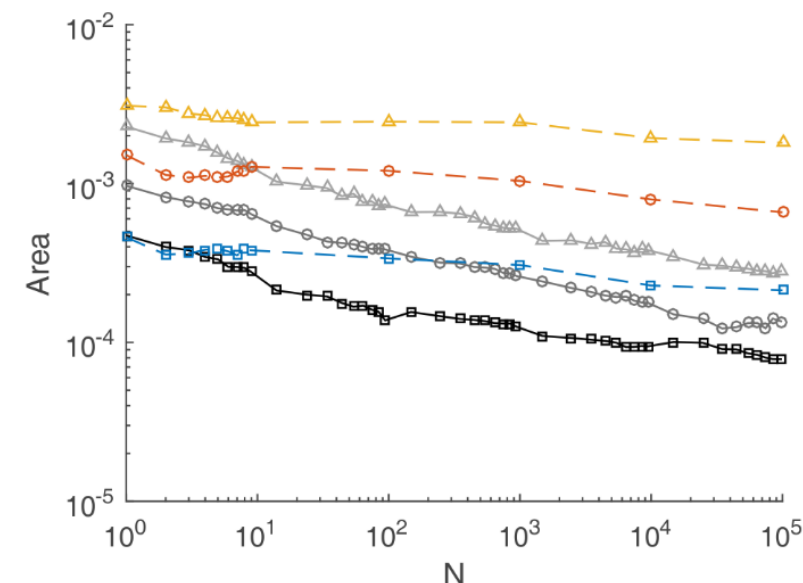

(b)

\begin{tabular}{|c|c|}
\hline$\longrightarrow$ Test CMLT1 & - - - - HARM Prediction CMLT1 \\
\hline - & $-\theta-$ HARM Prediction CMLT2 \\
\hline$\triangle \triangle$ Test CMLT3 & $-\triangle-$ HARM Prediction CMLT3 \\
\hline
\end{tabular}

Figure 13. Prediction of the evolution of (a) the secant stiffness and (b) the hysteresis loop area for tests CMLT1,2 and 3

Where $\hat{k}_{0}$ is the initial kinematic hardening strengths (Equation 22) and $m_{k}$ is the exponent that defines the rate at which the hysteresis loop closes with hardening. If $m_{k}=0$, this effect is disabled and therefore the shape of the hysteresis loop remains unchanged. In this case, it is preferable to choose the ratcheting rate to be a function of $\hat{k}_{0}$ rather than $\hat{k}$ (Equation 21 ). 
Equation 39 is one of the many options that can be used to define the change in loop shape, and other hardening mechanisms could be used. Equation 39 modifies the demonstration from Appendix $A$ and therefore the mathematical developments shown in this paper. In addition, any change in the hysteresis loop shape through modification of $\hat{k}$ or $\widehat{H}$ implies that the accelerated form of the model is not exact and that further computational criteria must be introduced to enable acceleration of computation.

As the change in hysteresis loop shape is not a primary effect of the ratcheting behaviour here, and might be due to the specific conditions for testing in Abadie, Byrne [29] (e.g. low relative density), it was therefore chosen not to include Equation 39 in the final modelling phase. However, further information on calibration and results are developed in Abadie [21].

\section{Conclusion}

This paper demonstrates a methodology for calibration of the HARM model presented in [3] and the rationale behind the parameter values such as adopted in Abadie et al. 2017. Equation 30, Equation 31 and Equation 32 provide analytical expressions for calibration of the ratcheting parameters, solely based on the fit from one monotonic test and a few continuous cyclic loading tests (Equation 16 and Equation 17). This fit can be further improved via optimisation, with the analytical formulation used as initial value. Prediction of both continuous and multi-amplitude cyclic tests demonstrate high accuracy and fit to experimental data.

This approach provides insights to the response at high cycle number in a computationally efficient way, as well as the response and capacity on re-loading following long-term cyclic loading. This is vital for assessment of service life of offshore wind turbine foundations and should be of great benefit to industry. To calibrate the model, precision in the prediction of the backbone curve is required, and approaches such as that proposed by Byrne, McAdam [33] or Jeanjean, Zhang [34] may be used for monopile design. Further work on the link between cyclic element testing and the macro-behaviour of the pile (translated through Equation 17) will enable the model to be calibrated through conventional in-situ and laboratory testing for use in industrial design. 


\section{Acknowledgements}

We are grateful to EDF Energies Nouvelles and EDF R\&D as well as more recently $\emptyset$ rsted for their funding of this work, including the financial support of the first Author.

\section{Notation}

$d$

Eo $\quad-\quad$ Initial tangent shear modulus

$f \quad \mathrm{FL}^{-2} \quad$ Specific Helmholtz free energy

$H$

$H_{n,}, \widehat{H}$

$k_{n,}, \hat{k}$

$k_{U}$

$m_{\alpha}$

$m_{h}$

$m_{k}$

$m_{s}$

$m_{r}$

$N$

$N_{S}$

$R_{0}$

$R_{n}, \hat{R}$

$R_{f a c}$

$y, y_{t}$

$\alpha, \alpha_{n}, \hat{\alpha}$

$\alpha_{r}$

$\beta$

$\beta_{0}$

$\varepsilon$

$\varepsilon \mathrm{pU}$

Dissipation potential

Lateral force on top of the pile

Kinematic hardening modulus

Upper and Lower limit streee

Number of cycles

Initial ratcheting rate

Ratcheting parameter

Acceleration factor

Internal kinematic variable

Ratcheting strain

Strain
Kinematic hardening surface stengths

Empirical exponent defining evolution of accumulated rotation with cycle number Monotonic exponent defining shape of loading curve

Exponent defining rate at which the hysteresis loop closes with hardening parameter

Exponent defining dependence of rate of ratcheting on stress

Exponent defining dependence of rate of ratcheting on hardening parameter

Number of kinematic hardening surfaces

Pile displacement, Pile displacement at point of load application

Accumulation of ratcheting strain

Initial value of hardening parameter

Ultimate monotonic plastic strain defining shape of loading curve 


Modified/Generalised signum function $\begin{cases}S(x)=1, & x>0 \\ S(x)=-1, \vdots & x<0 \\ S(x) \in[-1,1], & x=0\end{cases}$

\section{References}

[1] Abadie CN, Byrne BW, Houlsby GT. Rigid pile response to cyclic lateral loading: laboratory tests. Géotechnique. 2018.

[2] Abadie CN, Byrne BW, Levy-Paing S. Model pile response to multi-amplitude cyclic lateral loading in cohesionless soils. 3rd International Symposium on Frontiers in Offshore Geotechnics (ISFOG). Oslo, Norway2015. p. 6816.

[3] Houlsby GT, Abadie CN, Beuckelaers WJAP, Byrne BW. A model for nonlinear hysteretic and ratcheting behaviour. International Journal of Solids and Structures. 2017;120(67-80.

[4] Armstrong PJ, Frederick CO. A mathematical representation of the multiaxial Bauschinger effect. In: 731 CRNRBN, editor.1966.

[5] Frederick CO, Armstrong PJ. A mathematical representation of the multiaxial Bauschinger effect. Materials at High Temperatures. 2007;24(1):1-26.

[6] Lemaitre J, Chaboche J-L. Mécanique des matériaux solides, 3rd edition2010.

[7] Ohno N, Wang J. On modelling of kinematic hardening for ratcheting behaviour. Nuclear Engineering and Design. 1995;153(2-3):205-12.

[8] Chaboche JL. On some modifications of kinematic hardening to improve the description of ratchetting effects. International Journal of Plasticity. 1991;7(7):661-78.

[9] Masing G. Eiganspannungen und Verfestigung beim Messing. Proceedings for the 2nd International Congress of Applied Mechanics1926.

[10] Reese LC, Cox WR, Koop FD. Analysis of laterrally loaded piles in sand. 6th Offshore Technology Conference. Houston 21974.

[11] O'Neill MW, Murchison JM. An evaluation of p-y relationships in cohesionless soils. In: GT-DF02-83 RRN, editor.: Departement of Civil Engineering, University of Houston, Texas, 1983.

[12] API. Recommended Practice for Planning, Designing and Constructing Fixed Offshore Platforms, RP2A-WSD, Washington. In: Institute AP, editor.2010.

[13] DNV. Offshore Standard DNV-OS-J101, Design of offshore wind turbine structures (last edition). 2014.

[14] Poulos HG. Single pile response to cyclic lateral load. Journal of Geotechnical and Geoenvironmental Engineering. 1982;108(355-75.

[15] Little R, Briaud J-L. Cyclic horizontal load tests on six piles in sands at Houston Ship Channel. Research report 5640 to USAE Waterways Experiment Station, Miscellanous paper GL-88-27. Civil Engineering Department, Texas A\&M University1988.

[16] Achmus M, Kuo YS, Abdel-Rahman K. Behavior of monopile foundations under cyclic lateral load. Computers and Geotechnics. 2009;36(725-35.

[17] Miner AM. Cumulative damage in fatigue. Journal of applied mechanics. 1945:159-65. 
[18] LeBlanc C, Byrne BW, Houlsby GT. Response of Stiff Piles to Random Two-Way Lateral Loading. Géotechnique. 2010;60(9):715-21.

[19] LeBlanc C, Houlsby GT, Byrne BW. Response of Stiff Piles in Sand to Long-term Cyclic Lateral Loading. Géotechnique. 2010;60(2):79-90

[20] Peralta P. Investigations on the Behavior of Large Diameter Piles under Long-Term Lateral Cyclic Loading in Cohesionless Soil [PhD]: University of Hannover, 2010.

[21] Abadie CN. Cyclic Lateral Loading of Monopile Foundations in Cohesionless Soils: University of Oxford, 2015.

[22] Ziegler $\mathrm{H}$. Some extremum principles in irreversible thermodynamics with applications to continuum mechanics. Prog Solid Mech. 1963;4(93-193.

[23] Ziegler H. An Introduction to Thermomechanics. North Holland, Amsterdam1977.

[24] Houlsby GT, Puzrin AM. Principles of Hyperplasticity: an Approach to Plasticity Theory Based on Thermodynamic Principles. London2006.

[25] Puzrin AM, Houlsby GT. Fundamentals of kinematic hardening hyperplasticity. International Journal of Solids and Structures. 2001;38(21):3771-94

[26] Puzrin AM, Houlsby GT. Rate-dependent hyperplasticity with internal functions. Journal of Engineering Mechanics. 2003;129(3):252-63.

[27] Pyke RM. Nonlinear soil models for irregular cyclic loadings. Journal Geotechnical Engineering Division, ASCE. 1979;105(GT6, Proc Paper, 14642):715-26.

[28] Beuckelaers WJAP, Houlsby GT, Burd HJ. A comparison of the series and parallel Masing-Iwan model in 2D. 9th NUMGE Conference on Numerical Methods in Geotechnical Engineering. Porto, Portugal 2018. p. 173-179.

[29] Abadie CN, Byrne BW, Houlsby GT. Modelling of monopile response to cyclic lateral loading in sand. 8th International Conference of Offshore Site Investigation and Geotechnics (SUT OSIG). London 2017. p. 1046-53.

[30] Lin SS, Liao JC. Permanent strains of piles in sand due to cyclic lateral loads. Journal of Geotechnical and Geoenvironmental Engineering. 1999;125(798-802.

[31] Verdure L, Garnier J, Levacher D. Lateral cyclic loading of single piles in sand. International journal of physical modelling in geotechnics. 2003;3(17-28.

[32] Puech A, Garnier J. Design of Piles Under Cyclic Loading: SOLCYP Recommendations: ISTE Ltd., 2017.

[33] Byrne BW, McAdam RA, Burd HJ, Houlsby GT, Martin CM, Beuckelaers WJAP, et al. PISA: New Design Methods for Offshore Wind Turbine Monopiles. 8th International Conference of Offshore Site Investigation and Geotechnics (SUT OSIG). London2017. p. 142-61.

[34] Jeanjean P, Zhang Y, Zakeri A, Andersen KH, Gilbert R, Senanayake AIMJ. A framework for monotonic $p$-y curves in clays. 8th International Conference of Offshore Site Investigation and Geotechnics (SUT OSIG). London2017. p. $108-41$ 


\section{Appendix A: Derivation of the closed form expression for the ratcheting strain (Equation}

25)

Substituting Equation 22 and Equation 24 into Equation 7, this provides an expression for the plastic strain for this particular shape of backbone curve, during the initial loading phase $\left(\beta \in\left[\beta_{0}, \beta_{p 0}\right]\right)$. $\hat{\alpha}=\frac{\sigma-\hat{k}}{\hat{H}}=\left(\sigma-k_{U} \frac{\eta}{N_{S}}\right) \frac{m_{h}\left(m_{h}-1\right)}{N_{S}} \frac{\varepsilon_{p U}}{k_{U}}\left(\frac{\eta}{N_{S}}\right)^{m_{h}-2}, \quad \eta \in\left[0, \frac{\sigma}{k_{U}} N_{S}\right]$ on initial loading only $\quad$ Equation 40

\section{A.1. Initial loading (cycle 0)}

On first loading:

$\dot{\beta}=\dot{\alpha}_{r}=\int_{0}^{N_{S}} \hat{R} \dot{\hat{\alpha}} d \eta=\int_{0}^{N_{S}} R_{0}\left(\frac{\beta}{\beta_{0}}\right)^{-m_{r}}\left(\frac{\sigma}{k_{U}}\right)^{m_{S}}\left(\frac{\hat{k}}{k_{U}}\right) \dot{\hat{\alpha}} d \eta$

Which can be re-arranged into:

$\left(\frac{\beta}{\beta_{0}}\right)^{m_{r}} \dot{\beta}=R_{0} \int_{0}^{\sigma N_{S} / k_{U}}\left(\frac{\sigma}{k_{U}}\right)^{m_{S}}\left(\frac{\eta}{N_{S}}\right) \dot{\hat{\alpha}} d \eta$

Equation 42

From Equation 40, we have:

$\dot{\hat{\alpha}}=\frac{\dot{\sigma}}{\hat{H}}=\dot{\sigma} \frac{m_{h}\left(m_{h}-1\right)}{N_{S}} \frac{\varepsilon_{p U}}{k_{U}}\left(\frac{\eta}{N_{S}}\right)^{m_{h}-2}$

Equation 43

Substitution in Equation 42 and integration then gives:

$\left(\frac{\beta}{\beta_{0}}\right)^{m_{r}+1}=1+\frac{\varepsilon_{p U} R_{0}}{\beta_{0}} \frac{\left(m_{h}-1\right)\left(m_{r}+1\right)}{m_{s}+m_{h}+1}\left(\frac{\sigma}{k_{U}}\right)^{m_{s}+m_{h}+1}$

Equation 44

Specifically, at peak load (Figure 3):

$\left(\frac{\beta_{p 0}}{\beta_{0}}\right)^{m_{r}+1}=1+\frac{\varepsilon_{p U} R_{0}}{\beta_{0}} \frac{\left(m_{h}-1\right)\left(m_{r}+1\right)}{m_{s}+m_{h}+1}\left(\frac{\sigma_{p}}{k_{U}}\right)^{m_{s}+m_{h}+1}$

\section{A.2. Subsequent cyclic loops}

(1) Unloading 
In the following, we suppose a continuous 1-way cyclic load from 0 to $\sigma_{p}>0$. For any subsequent unload, the increment of ratcheting strain is (Equation 9 and Equation 20):

$\dot{\beta}=\dot{\alpha}_{r}=-\int_{0}^{N_{S}} \hat{R} \dot{\hat{\alpha}} d \eta=-\int_{0}^{N_{S}} R_{0}\left(\frac{\beta}{\beta_{0}}\right)^{-m_{r}}\left(\frac{\sigma}{k_{U}}\right)^{m_{S}}\left(\frac{\hat{k}}{k_{U}}\right) \dot{\hat{\alpha}} d \eta$

Equation 46

Which can be re-arranged into:

$\left(\frac{\beta}{\beta_{0}}\right)^{m_{r}} \dot{\beta}=-R_{0} \int_{0}^{\left(\sigma_{p}-\sigma\right) N_{S} / 2 k_{U}}\left(\frac{\sigma}{k_{U}}\right)^{m_{S}}\left(\frac{\eta}{N_{S}}\right) \dot{\hat{\alpha}} d \eta$

Equation 47

Substituting Equation 43 and integrating gives:

$\left(\frac{\beta}{\beta_{0}}\right)^{m_{r}} \dot{\beta}=-R_{0}\left(\frac{\sigma}{k_{U}}\right)^{m_{s}}\left(m_{h}-1\right) \frac{\varepsilon_{p U}}{k_{U}}\left(\frac{\sigma_{p}-\sigma}{2 k_{U}}\right)^{m_{h}} \dot{\sigma}$

Equation 48

We denote the current value of the state of load as $\sigma^{*}$. Integration from $\sigma_{p}$ to $\sigma^{*}$ gives:

$$
\begin{aligned}
\frac{\beta_{0}}{m_{r}+1}\left[\left(\frac{\beta}{\beta_{0}}\right)^{m_{r}+1}-\left(\frac{\beta_{p}}{\beta_{0}}\right)^{m_{r}+1}\right] & =\int_{\sigma_{p}}^{\sigma^{*}}-R_{0}\left(\frac{\sigma}{k_{U}}\right)^{m_{s}}\left(m_{h}-1\right) \frac{\varepsilon_{p U}}{k_{U}}\left(\frac{\sigma_{p}-\sigma}{2 k_{U}}\right)^{m_{h}} d \sigma \\
& =\frac{R_{0}\left(m_{h}-1\right) \varepsilon_{p U}}{2^{m_{h}} k_{U}} \int_{\sigma^{*}}^{\sigma_{p}}\left(\frac{\sigma}{k_{U}}\right)^{m_{s}}\left(\frac{\sigma_{p}-\sigma}{k_{U}}\right)^{m_{h}} d \sigma \\
& =\frac{R_{0}\left(m_{h}-1\right) \varepsilon_{p U}}{2^{m_{h}}}\left(\frac{\sigma_{p}}{k_{U}}\right)^{m_{s}+m_{h}+1 \sigma_{p}} \int_{\sigma^{*}}\left(\frac{\sigma}{\sigma_{p}}\right)^{m_{s}}\left(\frac{\sigma_{p}-\sigma}{\sigma_{p}}\right)^{m_{h}} \frac{d \sigma}{\sigma_{p}} \\
& =\frac{R_{0}\left(m_{h}-1\right) \varepsilon_{p U}}{2^{m_{h}}}\left(\frac{\sigma_{p}}{k_{U}}\right)^{m_{s}+m_{h}+1} \int_{x}^{1} t^{m_{s}}(1-t)^{m_{h}} d t
\end{aligned}
$$

Equation 49

Where $t=\sigma / \sigma_{p}, x=\sigma^{*} / \sigma_{p}$.

For $\sigma^{*}=0$ this reduces to a Beta function $B(.,$.$) :$

$\frac{\beta_{0}}{m_{r}+1}\left[\left(\frac{\beta}{\beta_{0}}\right)^{m_{r}+1}-\left(\frac{\beta_{p}}{\beta_{0}}\right)^{m_{r}+1}\right]=\frac{R_{0}\left(m_{h}-1\right) \varepsilon_{p U}}{2^{m_{h}}}\left(\frac{\sigma_{p}}{k_{U}}\right)^{m_{s}+m_{h}+1} \mathrm{~B}\left(m_{s}+1, m_{h}+1\right)$

Equation $\mathbf{5 0}$

Which rearranges for $\sigma^{*}=\sigma_{m}=0$ as:

$\beta_{m}^{m_{r}+1}=\beta_{p}^{m_{r}+1}+\frac{\beta_{0}^{m_{r}} R_{0}\left(m_{h}-1\right)\left(m_{r}+1\right) \varepsilon_{p U}}{2^{m_{h}}}\left(\frac{\sigma_{p}}{k_{U}}\right)^{m_{s}+m_{h}+1} \mathrm{~B}\left(m_{s}+1, m_{h}+1\right)$

Equation 51

(2) Re-loading 
On re-loading, we have:

$\dot{\beta}=\dot{\alpha}_{r}=\int_{0}^{N_{S}} \hat{R} \dot{\hat{\alpha}} d \eta=\int_{0}^{N_{S}} R_{0}\left(\frac{\beta}{\beta_{0}}\right)^{-m_{r}}\left(\frac{\sigma}{k_{U}}\right)^{m_{S}}\left(\frac{\hat{k}}{k_{U}}\right) \dot{\hat{\alpha}} d \eta$

Equation 52

Substituting Equation 43 and integrating from $\sigma_{m}$ to $\sigma^{*}$ gives in this case:

$\frac{\beta_{0}}{m_{r}+1}\left[\left(\frac{\beta}{\beta_{0}}\right)^{m_{r}+1}-\left(\frac{\beta_{m}}{\beta_{0}}\right)^{m_{r}+1}\right]=\frac{R_{0}\left(m_{h}-1\right) \varepsilon_{p U}}{2^{m_{h}}} \int_{1}^{x} t^{m_{s}}(t-1)^{m_{h}} d t$

Equation 53

In the specific case of 1-way loading $\left(\sigma_{m}=0\right)$, this reduces to the simpler form:

$\beta^{m_{r}+1}=\beta_{m}^{m_{r}+1}+\frac{\beta_{0}^{m_{h}} R_{0}\left(m_{h}-1\right)\left(m_{r}+1\right) \varepsilon_{p U}}{2^{m_{h}}\left(m_{s}+m_{h}+1\right)}\left(\frac{\sigma^{*}}{k_{U}}\right)^{m_{s}+m_{h}+1}$

Equation 54

Which hence gives the value of $\beta_{p}$ at peak load.

\section{A.3. Equation for calibration}

Putting together the solutions for unloading and reloading in the first cycle, we get:

$\beta_{p 0}^{m_{r}+1}=\beta_{0}^{m_{r}+1}+\beta_{0}^{m_{r}} \varepsilon_{p U} R_{0} \frac{\left(m_{h}-1\right)\left(m_{r}+1\right)}{m_{s}+m_{h}+1}\left(\frac{\sigma_{p}}{k_{U}}\right)^{m_{s}+m_{h}+1}$

Equation 55

$\beta_{m 1}^{m_{r}+1}=\beta_{p 0}^{m_{r}+1}+\frac{\beta_{0}^{m_{r}} R_{0}\left(m_{h}-1\right)\left(m_{r}+1\right) \varepsilon_{p U}}{2^{m_{h}}} \mathrm{~B}\left(m_{s}+1, m_{h}+1\right)$

Equation 56

$$
\begin{aligned}
\beta_{p 1}^{m_{r}+1} & =\beta_{m 1}^{m_{r}+1}+\frac{\beta_{0}^{m_{r}} R_{0}\left(m_{h}-1\right)\left(m_{r}+1\right) \varepsilon_{p U}}{2^{m_{h}}\left(m_{s}+m_{h}+1\right)}\left(\frac{\sigma_{p}}{k_{U}}\right)^{m_{s}+m_{h}+1} \\
& =\beta_{p 0}^{m_{r}+1}+\frac{\beta_{0}^{m_{r}} R_{0}\left(m_{h}-1\right)\left(m_{r}+1\right) \varepsilon_{p U}}{2^{m_{h}}}\left(\frac{\sigma_{p}}{k_{U}}\right)^{m_{s}+m_{h}+1}\left(\mathrm{~B}\left(m_{s}+1, m_{h}+1\right)+\frac{1}{m_{s}+m_{h}+1}\right)
\end{aligned}
$$

Equation 57

By recurrence of the above procedure, we then have the remarkable result that $\beta_{p N}^{m_{r}+1}$ simply accumulates linearly with $N$ :

$\beta_{p N}^{m_{r}+1}=\beta_{p 0}^{m_{r}+1}+N \frac{\beta_{0}^{m_{r}} R_{0}\left(m_{h}-1\right)\left(m_{r}+1\right) \varepsilon_{p U}}{2^{m_{h}}}\left(\frac{\sigma_{p}}{k_{U}}\right)^{m_{s}+m_{h}+1}\left(\mathrm{~B}\left(m_{s}+1, m_{h}+1\right)+\frac{1}{m_{s}+m_{h}+1}\right)$

Equation 58

Substituting Equation 45 and using the notations of Equation 26, Equation 27 and Equation 28, this leads to Equation 25. 


\section{A.4. Case of Equation 19}

If one prefers to use Equation 19 instead of Equation 20 for $\hat{R}$, then Equation 41 simply becomes:

$\dot{\beta}=\dot{\alpha}_{r}=\int_{0}^{N_{S}} \hat{R} \dot{\hat{\alpha}} d \eta=\int_{0}^{N_{S}} R_{0}\left(\frac{\beta}{\beta_{0}}\right)^{-m_{r}}\left(\frac{\sigma}{k_{U}}\right)^{m_{S}} \dot{\hat{\alpha}} d \eta$

Equation 59

Equation 43 remains unchanged and this leads to Equation 44 becoming:

$\left(\frac{\beta}{\beta_{0}}\right)^{m_{r}+1}=1+\frac{\varepsilon_{p U} R_{0}}{\beta_{0}} \frac{m_{h}\left(m_{r}+1\right)}{m_{s}+m_{h}}\left(\frac{\sigma}{k_{U}}\right)^{m_{s}+m_{h}}$

Equation 60

Similarly, Equation 51 and Equation 54 become:

$\beta_{m}^{m_{r}+1}=\beta_{p}^{m_{r}+1}+\frac{\beta_{0}^{m_{r}} R_{0} m_{h}\left(m_{r}+1\right) \varepsilon_{p U}}{2^{m_{h}-1}}\left(\frac{\sigma_{p}}{k_{U}}\right)^{m_{s}+m_{h}} \mathrm{~B}\left(m_{s}+1, m_{h}\right)$

Equation 61

$\beta^{m_{r}+1}=\beta_{m}^{m_{r}+1}+\frac{\beta_{0}^{m_{h}} R_{0} m_{h}\left(m_{r}+1\right) \varepsilon_{p U}}{2^{m_{h}-1}\left(m_{s}+m_{h}\right)}\left(\frac{\sigma^{*}}{k_{U}}\right)^{m_{s}+m_{h}}$

Equation 62

This leads to Equation 33. 